\title{
COMMENTARY
}

ROLE OF THE VITREOUS IN THE GAUSATION OF GAVERNOUS ATROPHY

Cause of cavernous atrophy of the optic nerve

It appears that the primary cause of cavernous degeneration is infarction of the circulation in the optic nerve. Changes then occur which are analogous to the lacunar infarcts which Pierre Marie described in the basal ganglia. In chronic glaucoma this process goes back in the optic nerve. Histologically there is no reaction with microglia or macrophages. If the pressure is high, as in angle-closure glaucoma, an ischaemic infarct occurs in which there is a proliferation of glial cells and macrophages and scarring with degeneration of the nerve fibres. Later, when the nerve fibres have degenerated, small openings remain in the lamina cribrosa through which larger molecules can be forced backwards under high pressure. It is thought that this is how the vitreous comes to be forced into the optic nerve so that the septa previously occupied by nerve fibres become distended with acid mucopolysaccharide. The supporting connective tissue does not undergo degeneration as it is able to withstand a very much higher intraocular pressure.

On the experimental evidence available to him, Mr. S. S. Hayreh (see p. I84) felt that the vitreous was unlikely to pass through the lamina cribrosa, as he had never been able to find any trace of colloidal iron in the optic disc or nerve after it had been injected into the vitreous, however high the pressure was raised.

\section{Aqueous outflow pathway in normal and glaucomatous eyes}

\author{
RAMESH C. TRIPATHI \\ Department of Pathology, Institute of Ophthalmology, University of London
}

The discovery by Schlemm (1830-31) of a circular canal in the angular region of the human eye has led to extensive investigations being carried out to establish its structure and function in relation to the drainage of the aqueous humour. There is little doubt at present as to the free flow of the aqueous through the intercommunicating spaces of the trabecular meshwork. However, the question whether or not the endothelial lining cf Schlemm's canal constitutes a structural barrier to the aqueous outflow has been debated for well over a century. We remain especially indebted to Professor Norman Ashton, who clarified the anatomical relations of Schlemm's canal and associated structures by elegant injection models and painstaking studies of their microstructure (Ashton, 1952, 1960; Ashton, Brini, and Smith, 1956).

Over the past decade, the application of electron microscopy has brought renewed interest in correlating the structure and function of the exit pathway of the aqueous. This report is mainly concerned with the presentation of my personal observations on the fine structure of the trabecular apparatus in relation to aqueous outflow in normotensive eyes of primates and lower mammals and with special reference to the pathological alterations in the angular region of some cases of primary glaucoma. 


\section{PRIMATE EYES}

The detailed structure and ultrastructure of Schlemm's canal in normotensive human and monkey eyes has already been reported (Tripathi, I968, I969a,b, I970, I97 Ia). It will suffice here to summarize the main points of this study.

Light and electron microscopy of epoxy-resin embedded sections reveal that normally the canal of Schlemm is lined by a thin but continuous single layer of endothelium; on the trabecular (inner) side of the canal many cells show giant vacuoles and prominent nuclei projecting into the lumen of the canal (Figs I, 2, 3). In contrast, the endothelial cells lining the corneo-scleral side of the canal are flatter and rarely show vacuoles. The septae which bridge or divide the canal are also lined by endothelium which may be of the vacuolated or non-vacuolated type according to their proximity to the trabecular or corneoscleral side of the canal, and it continues uninterrupted with them. Because of these septae and the varying shape of the canal, it is difficult in some places to delineate the socalled inner and outer walls of Schlemm's canal merely by their anatomical disposition, and this raises the question whether the term canal is the most appropriate description for this structure (Tripathi, I969a).

The endothelial cells lining the canal are generally spindle-shaped, their long axis being parallel to that of the canal and, on the trabecular side, they measure $40-100 \mu \mathrm{m}$ in length and have a diameter of $5^{-12} \mu \mathrm{m}$ in the central region. Adjacent cells are joined by occluding zonules which occupy only a small area of the cell surface. Among the intracellular content the usual cell components, such as nucleus, mitochondria, Golgi

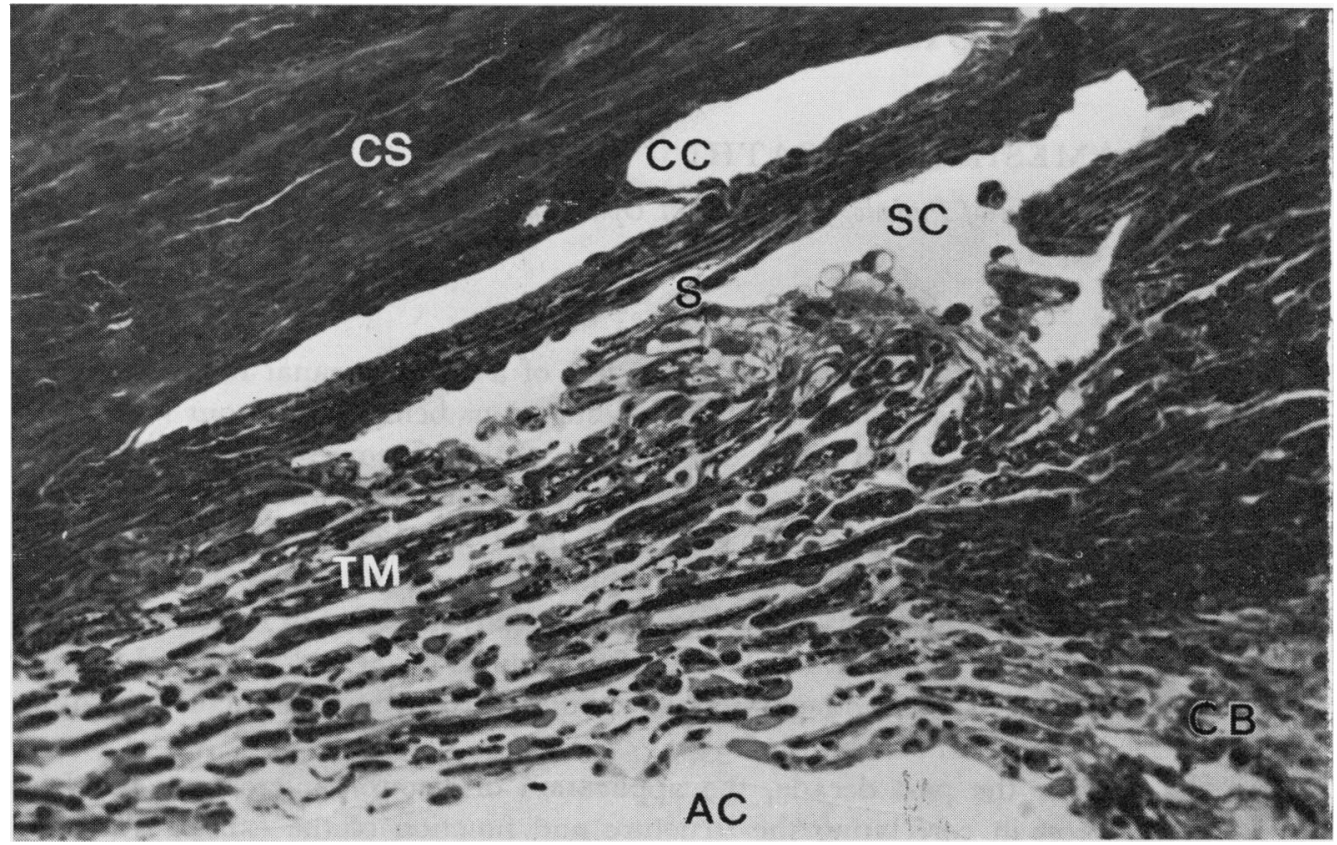

FIG. I Canal of Schlemm $(S C)$ and trabecular meshwork (TM) in anterior posterior section, showing undulating trabecular wall and septae $(S) . \quad C C=$ Collector channels. $C B=$ Ciliary body. $C S=$ Corneosclera. $A C=$ Anterior chamber. Osmium fixed, Araldite embedded, toluidine blue stained I $\mu$ thick section. Normotensive human eye, 55 years. Light photomicrograph. $\quad \times 180$ 


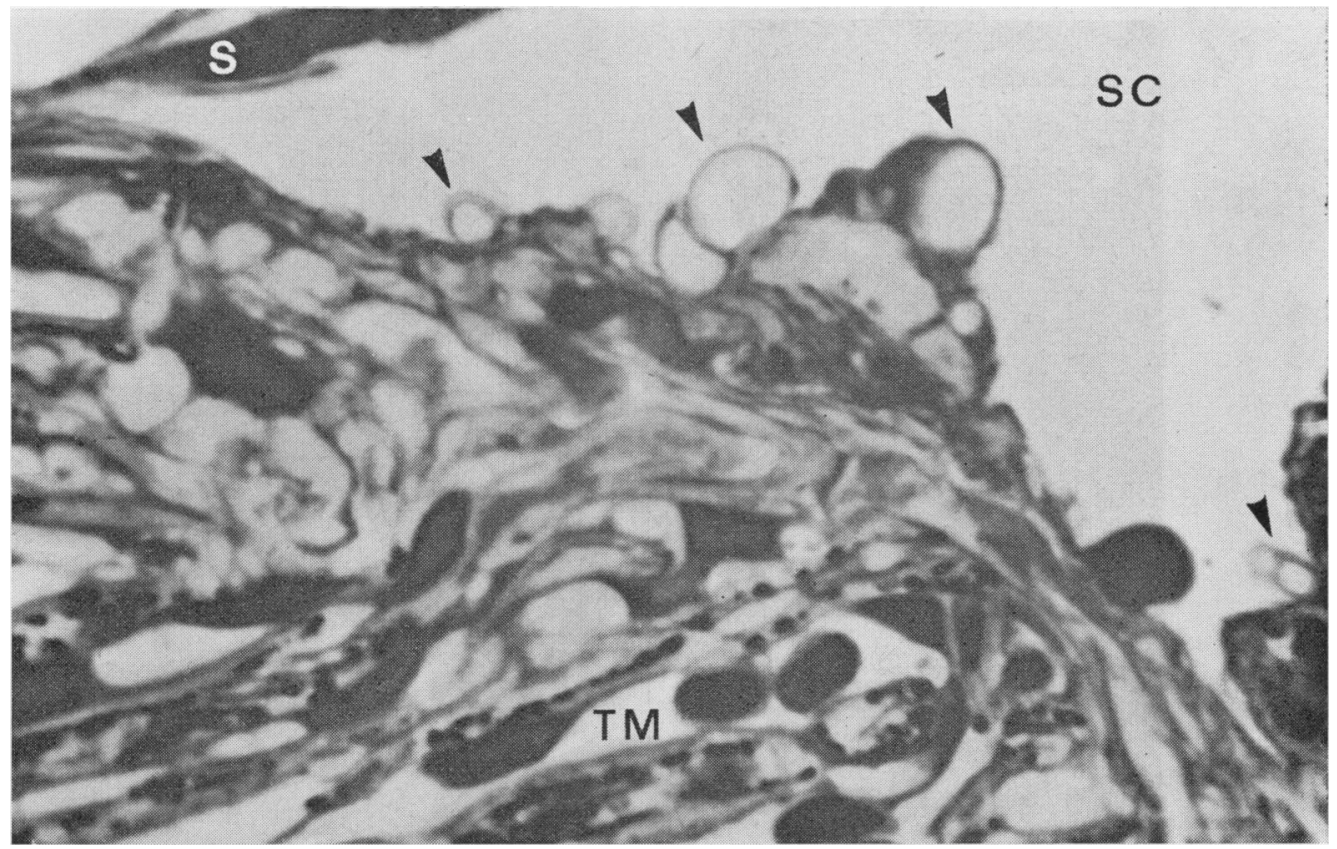

FIG. 2 High-power view of trabecular wall of Schlemm's canal $(S C)$, showing vacuolated endothelial celln (arrowed) projecting into the lumen of canal. $S=$ Septum. TM= Trabecular meshwork. Same sectios as Fig. 1. $\quad \times 1,260$

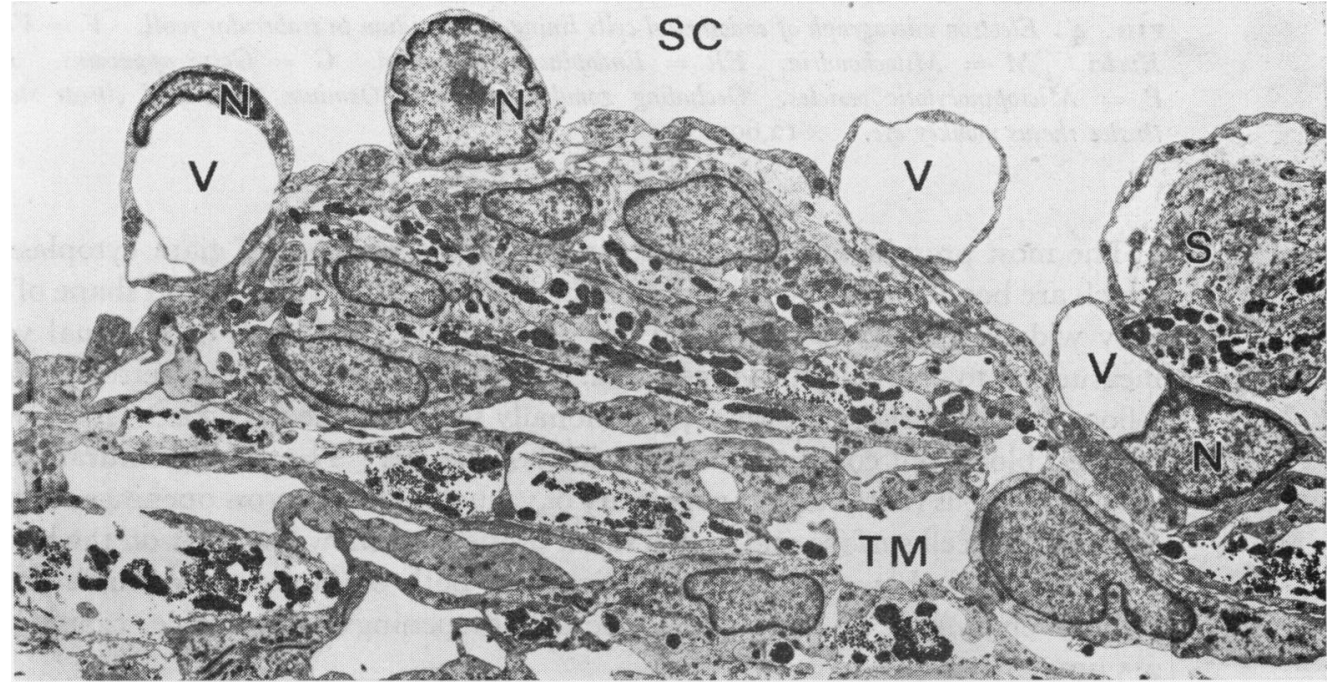

FIG. 3 Survey electron micrograph of trabecular wall of Schlemm's canal $(S C)$, showing giant vacuoles $(V)$ and nuclei $(\mathcal{N})$ projecting into lumen of canal. $S=$ Septum. $T M=$ Trabecular meshwork. Osmium fixed phosphotungstic acid stained. Normotensive human eye, 80 years. Approx. $\quad \times 3,850$

apparatus, endoplasmic reticulum, small vesicles, lysosomal bodies, centrioles, free glycogen, and ribonucleic acid granules, can easily be identified (Fig. 4 overleaf). Occasionally, one can also find fibrillar structures (approximately $7 \mu \mathrm{m}$ in diameter). 


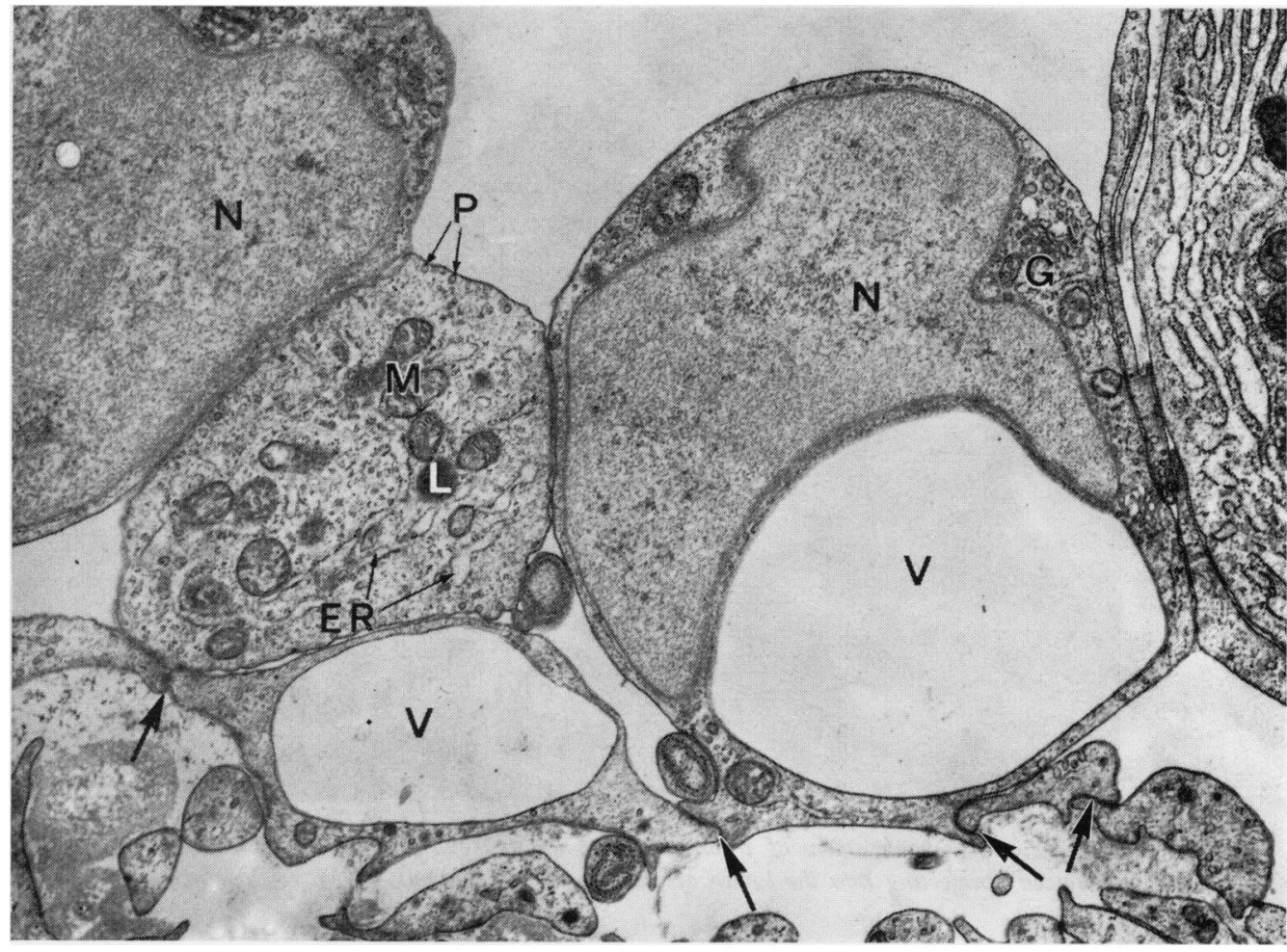

FIG. 4 Electron micrograph of endothelial cells lining diverticulum in trabecular wall. $\quad V=$ Vacuoles. $\quad \mathcal{N}=$ Nuclei. $\quad M=$ Mitochondria. $E R=$ Endoplasmic reticulum. $G=$ Golgi apparatus. $L=L$ Lysosome $P=$ Micropinocytotic vesicles. Occluding zonules arrowed. Osmium fixed, lead citrate stained. Normotensive rhesus monkey ere. $\times 12,600$

The most prominent feature of these cells is the presence of giant cytoplasmic vacuoles which are bound by a smooth unit membrane (Fig. 4). The size and shape of the vacuoles vary widely; diameters up to $2-6 \mu \mathrm{m}$ are common, although exceptional vacuoles may measure up to $20 \mu \mathrm{m}$ in the longest axis. Almost all vacuoles are electron optically empty; a flocculent material is, however, occasionally seen in some vacuoles. In some instances a trapped blood cell could also be seen in the vacuole. The ultrastructural analyses of the vacuolated cells reveal that the majority of vacuoles have narrow openings on the trabecular aspect of the cell surface and only a small proportion show openings on the luminal aspect; some vacuoles, however, show openings on both surfaces, thus constituting an intracellular channel. The size and shape of the openings are variable, ranging from 0.2 to $2 \cdot 5 \mu \mathrm{m}$.

To trace the exit pathway of the aqueous across the endothelial barrier of Schlemm's canal, the anterior chambers of monkey eyes were perfused in vivo with an electron dense tracer (ferritin serum solution) and analysed by light and electron microscopy. The majority of the vacuoles are filled with the tracer material through the basal openings (Figs 5 and 6); the cell junctions are intact and offer a barrier to the leakage of the tracer substance (Fig. 6). From some vacuoles ferritin material could be seen escaping into the lumen of Schlemm's canal through their luminal openings (Tripathi, r97 ra). 


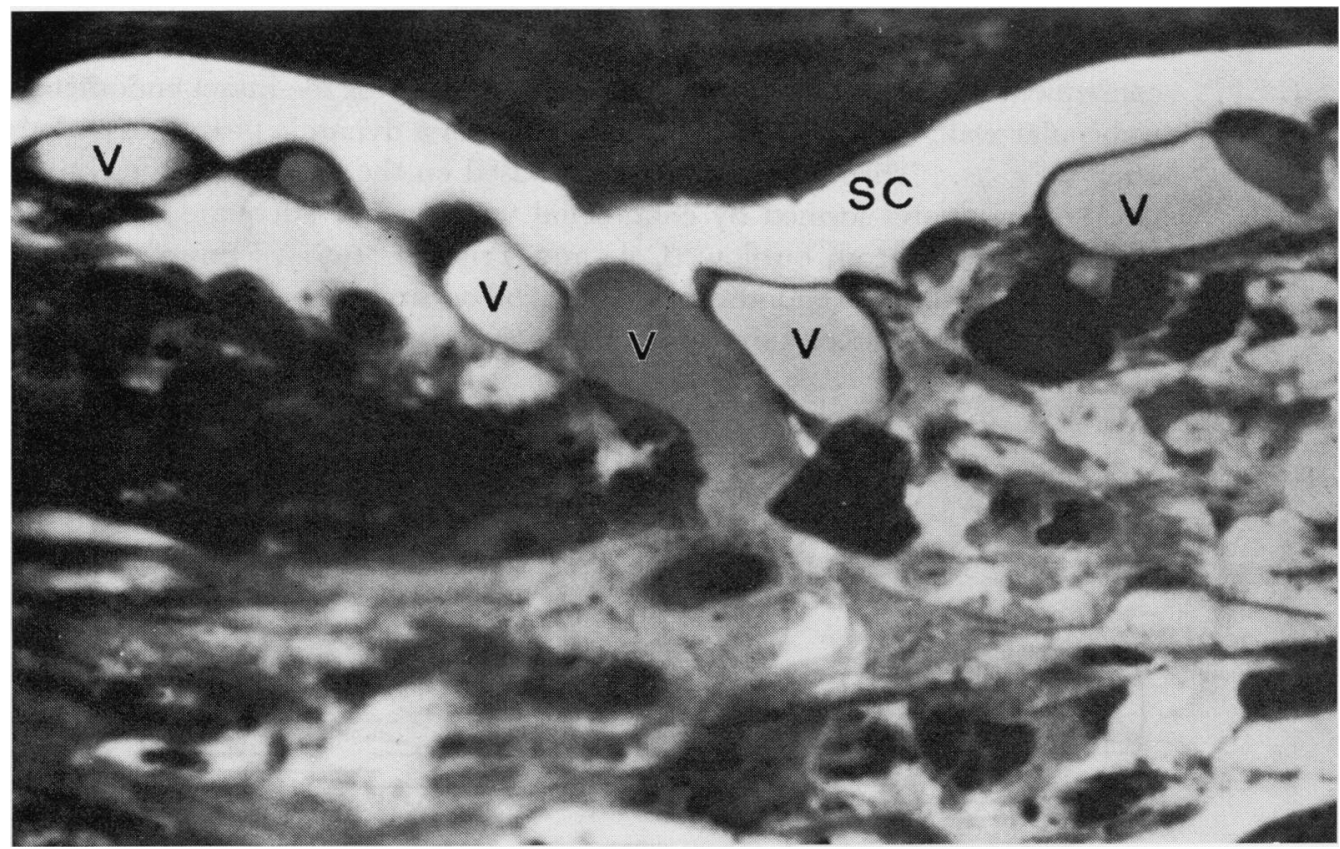

FIG. 5 Light photomicrograph of Schlemm's canal $(S C)$, showing giant vacuoles $(V)$ filled with injection material following an intracameral perfusion experiment at a controlled physiological intraocular pressure in rhesus monkey. After 20 minutes, the eye was fixed in vivo with osmium tetroxide. Araldite embedded, toluidine blue stained, I $\mu$ thick section. $\times 2,100$

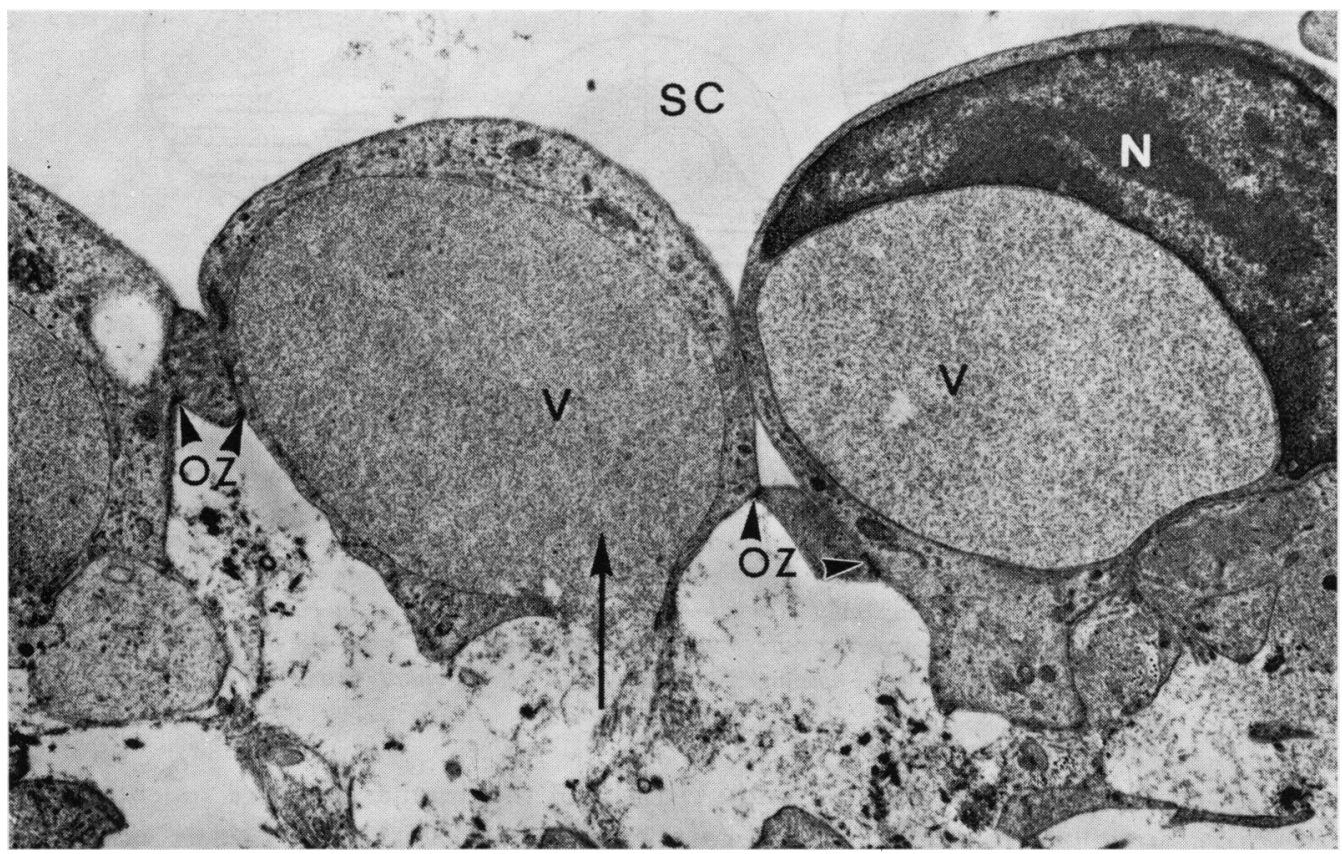

FI G. 6 Electron micrograph of endothelial cells lining trabecular wall of Schlemm's canal (SC), showing giant vacuoles $(V)$ filled with injection material. Arrow denotes basal opening of one vacuole. $O Z=$ Occluding zonules. $\quad \mathcal{N}=$ Nucleus. Same specimen as Fig. 5. Phosphotungstic acid stained. $\quad \times 10,500$ 
On the basis of these observations, the morphogenesis of the endothelial vacuoles, and consequently the outflow of the aqueous humour across the intact endothelial lining of the trabecular wall, is considered as dependent upon a dynamic process of endothelial vacuolation (Fig. 7). Theoretical calculations based on the data presently available show that the dynamic pores formed by endothelial vacuoles are adequate to provide the normal facility of the aqueous outflow (Cole and Tripathi, 197I). From this interpretation, it would appear that the endothelial vacuolation occurring normally in the wall of Schlemm's canal is an unique process since a similar mechanism has not been shown to exist elsewhere in the body.

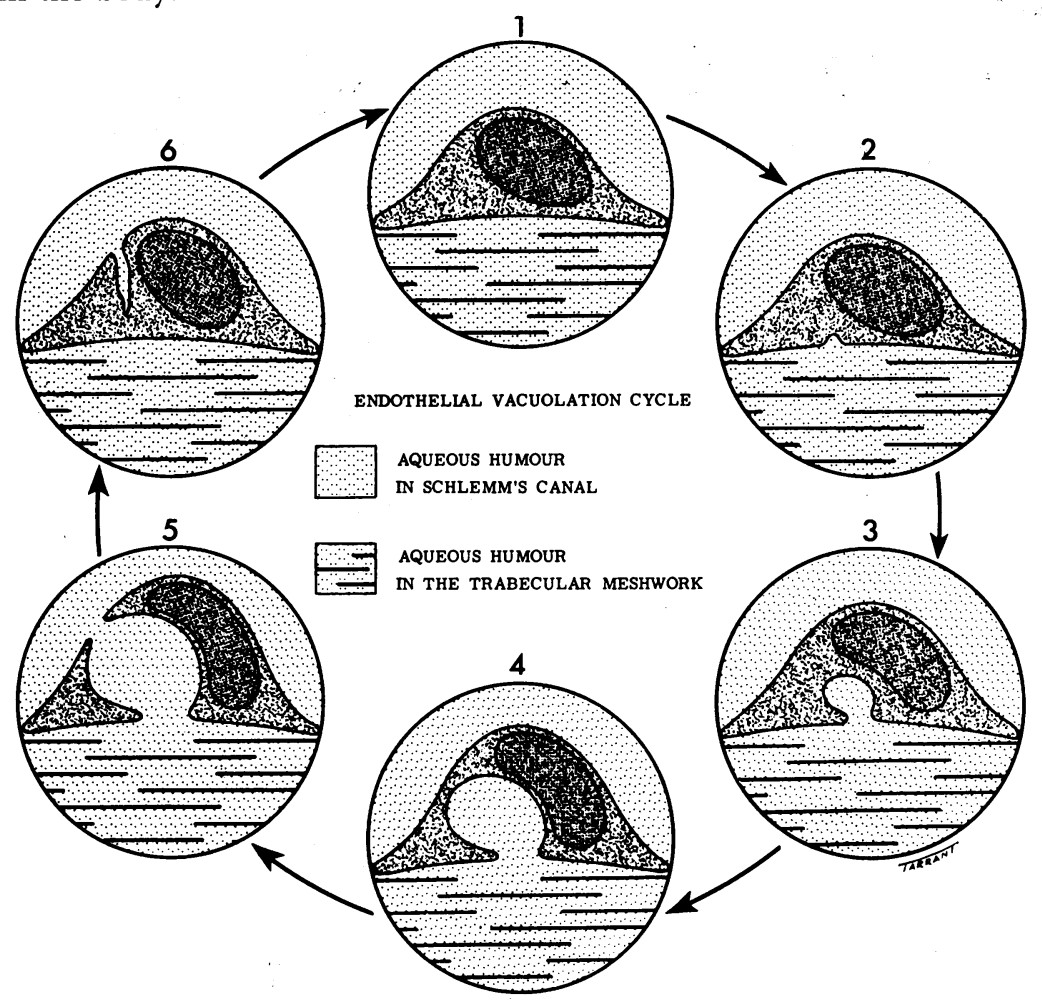

FIG. 7 Diagrammatic representation of endothelial vacuolation cycle and mechanism of aqueous outflow across 윽 trabecular wall of Schlemm's canal. The earliest vacuole (Stage 2) probably forms as an indentation or shallow $D$ depression on the basal aspect of the endothelial cell surface-macropinocytosis-a peculiar property of these cells, which may be partly dependent on the hydrostatic pressure gradient across the endothelial lining of the canal. $A \vec{N}$ giant vacuole is formed by the gradual enlargement of this invagination (Stages 3 and 4) and eventually ruptures into Schlemm's canal, thereby creating an intracellular channel (Stage 5). This stage, apart from providing an easier aqueous outflow, could also allow, in certain circumstances, a passage for particulate matter, blood cor- c心 puscles, and other wandering cells. After a certain interval of time, the cell cytoplasm moves towards the basal aspect to occlude the basal opening (Stage 6) and the cell eventually returns to its resting stage (Stage I)

\section{LOWER MAMMALS}

Preliminary studies (Tripathi, I97 Ib) of the angular tissue of rodentia (rabbit and rat), ungulata (ox and pig), and carnivora (cat and dog) clearly reveal that the channels concerned with the aqueous drainage, designated as "angular aqueous plexus" or simply as the "aqueous plexus", have a continuous endothelial lining, many cells of which show giant vacuoles (Figs 8 and 9). Many points of similarity exist between the angular 


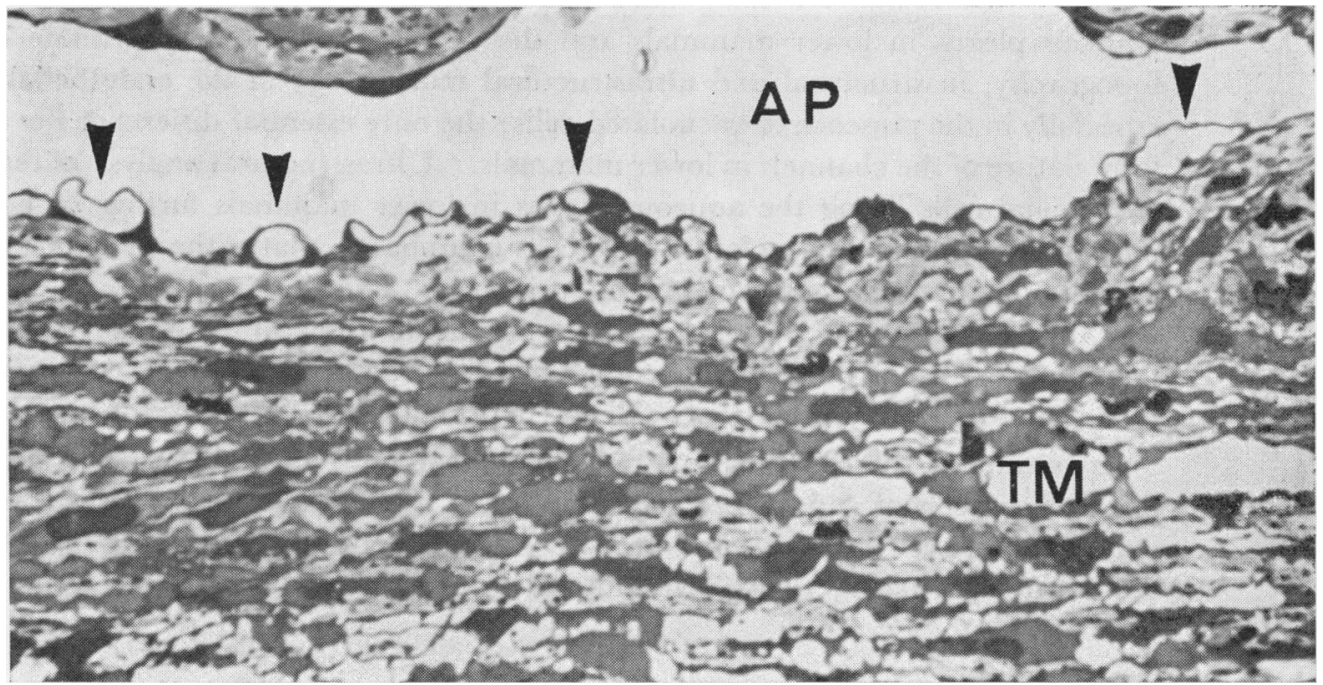

FIG. 8 Light photomicrograph of trabecular wall of angular aqueous plexus $(A P)$ in an ox eye. Arrows denote prominent vacuolated endothelial cells. TM=Trabecular meshwork. Osmium fixed, Araldite embedded, toluidine blue stained I $\mu$ thick section. $\quad \times 800$

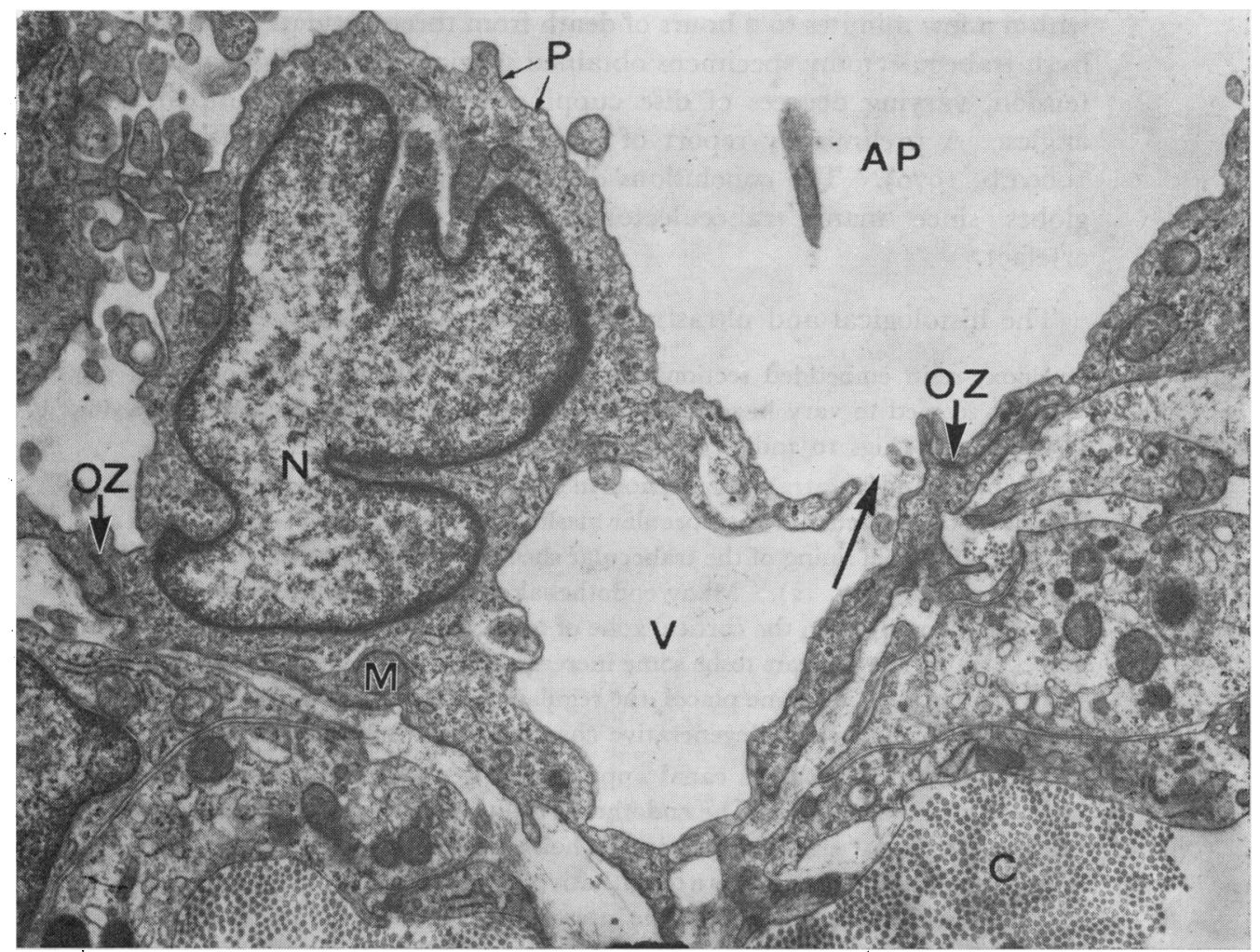

FIG. 9 Vacuolated endothelial cell lining the wall of the angular aqueous plexus $(A P)$ in a kitten eye. Note luminal opening (arrowed) of vacuole $(V) \quad \mathcal{N}=$ Nucleus. $\quad P=$ Micropinocytotic vesicles. $\quad M=$ Mitochondria. $\mathrm{OZ}=$ Occluding zonule. $C=$ Collagen fibrils. Osmium fixed, uranyl acetate and lead citrate stained. Electron micrograph. $\times 14,000$ 
aqueous plexus in lower mammals and the canal of Schlemm in primate eyes, e.g. in topography, in structural and ultrastructural morphology of the endothelial lining, and especially in the presence of vacuolated cells; the only essential difference lies in the plexiform nature of the channels in lower mammals. Ultrastructural analysis of the vacuolated endothelial cells lining the aqueous plexus in lower mammals further suggests that the morphogenesis of the vacuoles in these cells is similar to that of the vacuolated endothelial cells lining the canal of Schlemm in primates. This interpretation, therefore, entails that the functional porosity of the intact endothelial lining of the aqueous plexus in lower mammals is normally dependent on the dynamic process of endothelial vacuolation similar to that postulated for the canal of Schlemm in primate eyes (Tripathi, I969a, 1970, I 97 I a,b).

In summary, therefore, we may conclude that normally the aqueous outflow by the dynamic process of endothelial vacuolation is a fundamental mechanism common to the eyes of both lower mammals and primates and may possibly exist in the eyes of many other vertebrates (Tripathi, 1972; Tripathi and Tripathi, 1972).

\section{Glaucomatous eyes}

\section{CHRONIC SIMPLE GLAUCOMA}

This report is based on the electron microscopical study of six glaucomatous eyes enucleated within a few minutes to 2 hours of death from three patients aged 70 to 82 years and many fresh trabeculectomy specimens obtained surgically. All the patients had bilateral raised tension, varying degrees of disc cupping and field defect, impaired outflow, and open angles. A preliminary report of some of these cases has been given earlier (Tripathi, 1969a,b, 1970). The conclusions derived herein are mainly based on the study of intact globes since many trabeculectomy specimens were associated with some traumatic artefact.

The histological and ultrastructural findings are as follows:

In epoxy-resin embedded sections, almost identical changes were observed in all eyes, and these changes seemed to vary in severity rather than in nature depending on the stage of advancement of the disease (Figs io and $\mathrm{I}$ i).

The inter- and intratrabecular spaces in these eyes appear generally open or only slightly narrowed. The uveal component of the trabecular meshwork is well developed. There is an increased tendency for the endothelial lining of the trabecular sheets to be interrupted, separated from the cortical zone, and degenerate (Fig. I2). Many endothelial cells show aggregates of phagocytosed dense granule (pigment deposit). In the cortical zone of some trabecular sheets (more so in the uveal than scleral trabeculae), there appears to be some increase in the basement membrane material and "lattice" or "curly" collagen. In some places, the regular collagen fibrils and elastic components in and around the trabecular core show degenerative changes, together with an increase in "curly" collagen.

The lumen of Schlemm's canal appears slightly narrowed although its meridional dimension is normal (Figs Io and I I). The endothelial cells lining the canal are attenuated and have flattened nuclei. The most significant changes, however, are located in the trabecular wall of the canal (Figs I 3,14 , and I 5 ). There is a quantitative and qualitative depletion of giant vacuoles, most marked in advance stages of glaucoma. The intercellular junctions are intact and there is a reduced number of micropinocytotic vesicles. The basement membrane is relatively thicker and more continuous than normally seen. Beneath the endothelial lining, there is an increase in the extracellular fibrous, granular, and amorphous elements, with a decrease in the cellular component. In the corneoscleral wall, the endothelial lining appears atrophic in places and the supporting connective tissue zone shows an increase in amorphous, granular, and banded fibrous elements. 


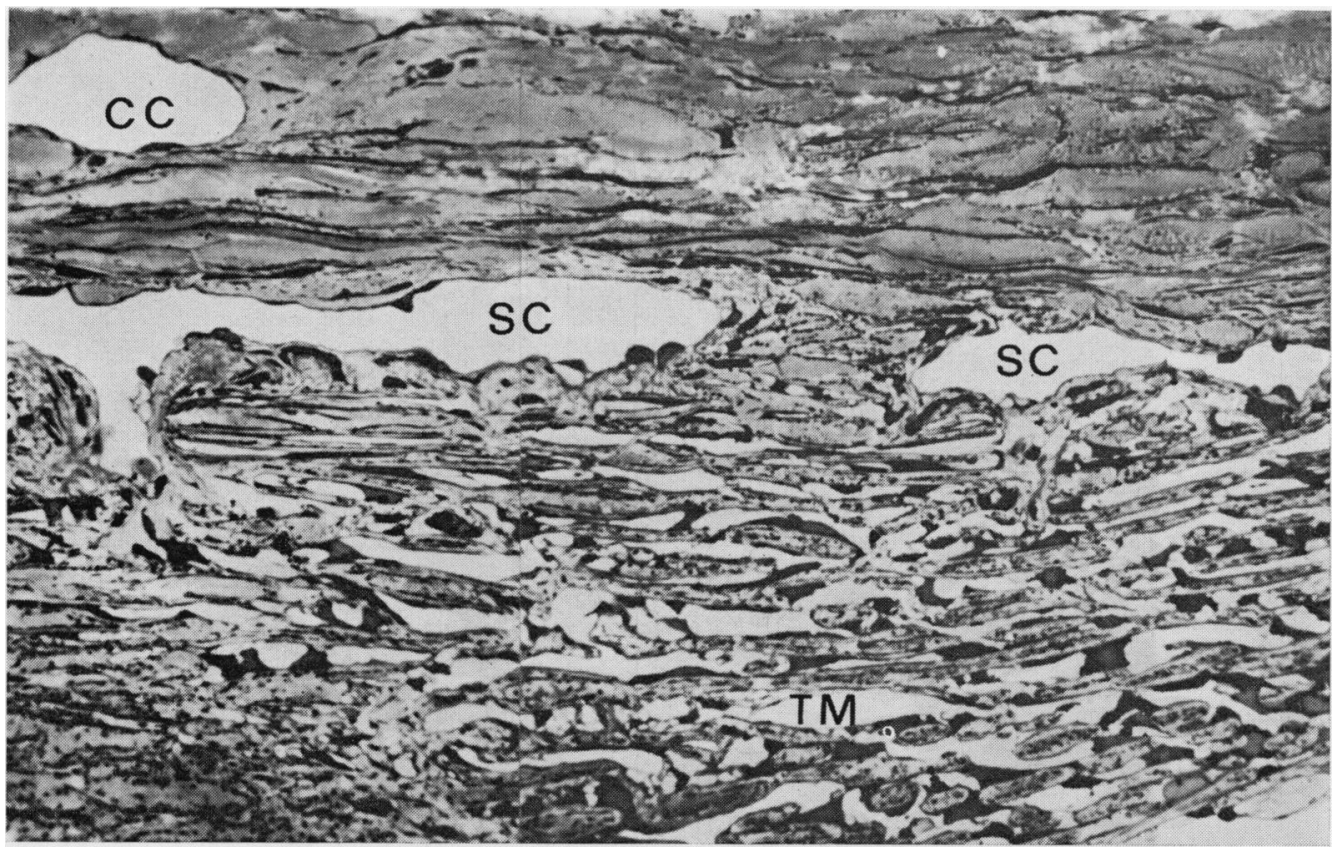

FIG. Io Meridional section of canal of Schlemm (SC) and trabecular meshwork (TM) in a 70-year-old man with moderately advanced simple glaucoma. In the endothelial lining of the canal, a few vacuolated cells can be seen. $C C=$ Collector channels. Glutaraldehydelosmium fixed, Epon embedded, toluidine blue stained, I $\mu$ thick section. Light photomicrograph. $\times 310$

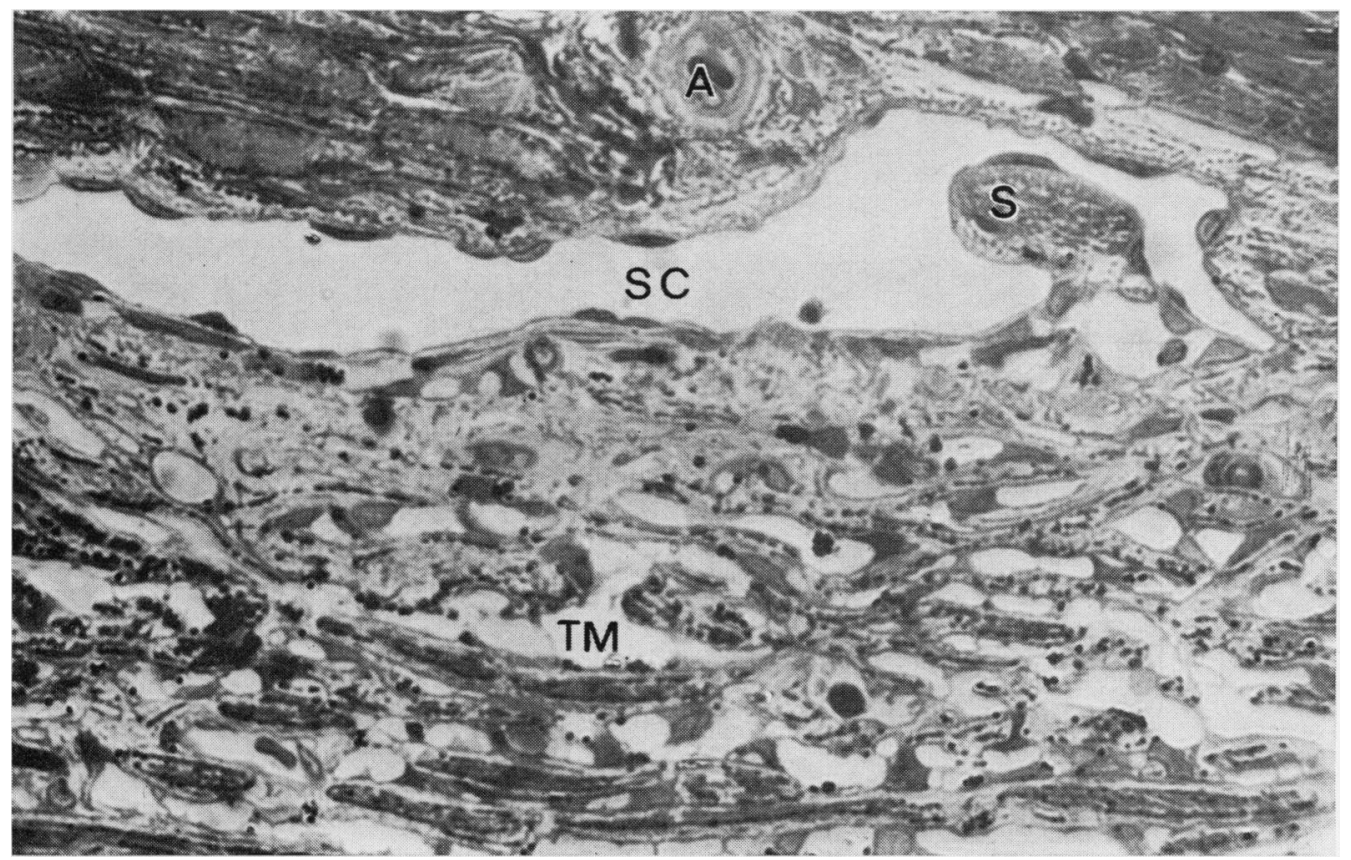

FIG. I I Meridional section of canal of Schlemm (SC) and trabecular meshwork (TM) in an 8o-year-old man with an advanced stage of simple glaucoma. The endothelial lining of the trabecular wall shows complete absence of giant vacuoles. $S=$ Septum in canal. $A=$ Arteriolar twig. Osmium fixed, Araldite embedded, toluidine blue stained I $\mu$ thick section. Light photomicrograph. $\quad \times 63^{0}$ 


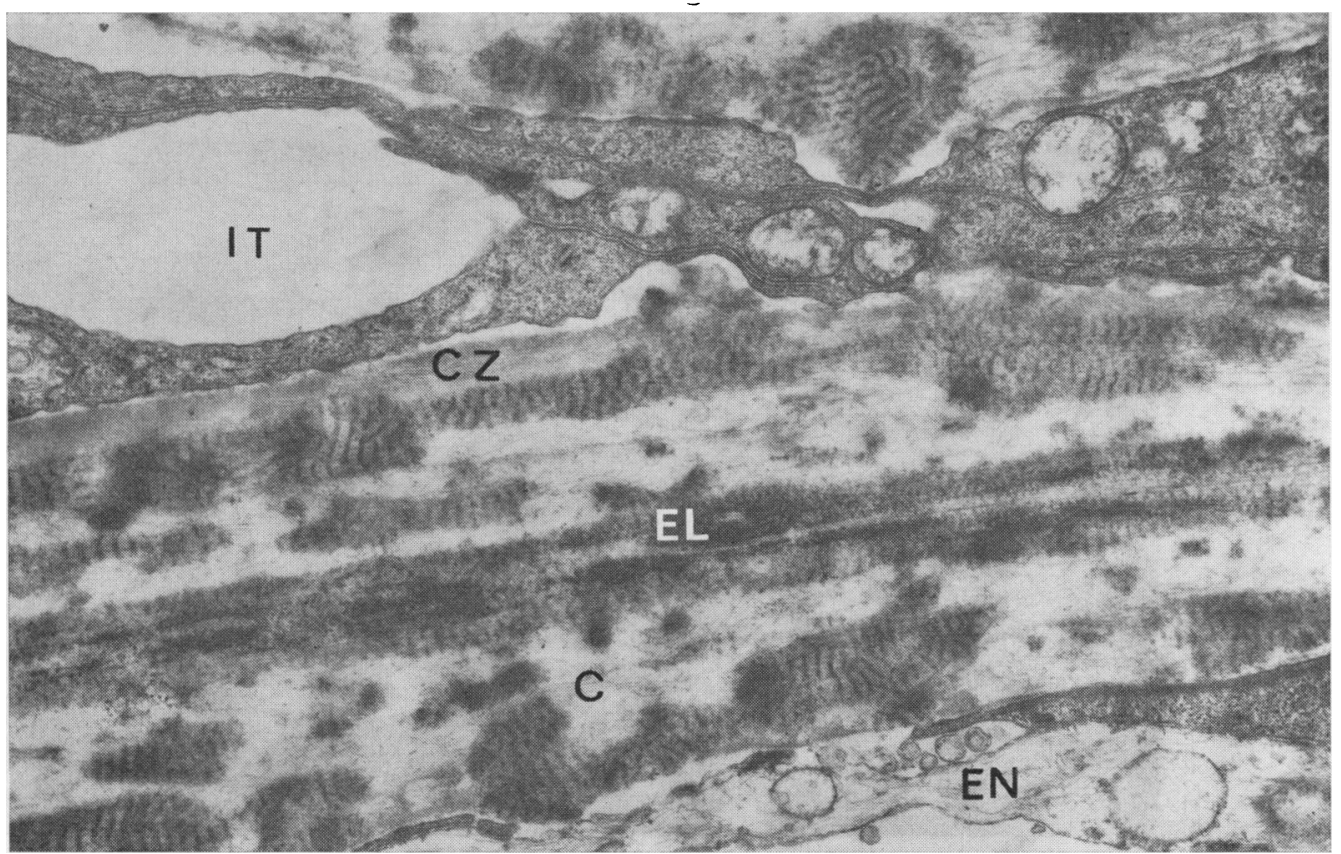

FIG. 12 Electron micrograph of trabecular sheet, showing degenerative changes in trabecular endothelium (EN), a thickened cortical zone (CZ) containing "curly" collagen, collagen fibrils $(C)$ in central core, and elastic element $(E L)$, the latter being associated with "curly" collagen. IT = Intertrabecular space. Uranyl acetate and lead citrate stained. Same case as Fig. $10 . \quad \times 14,000$

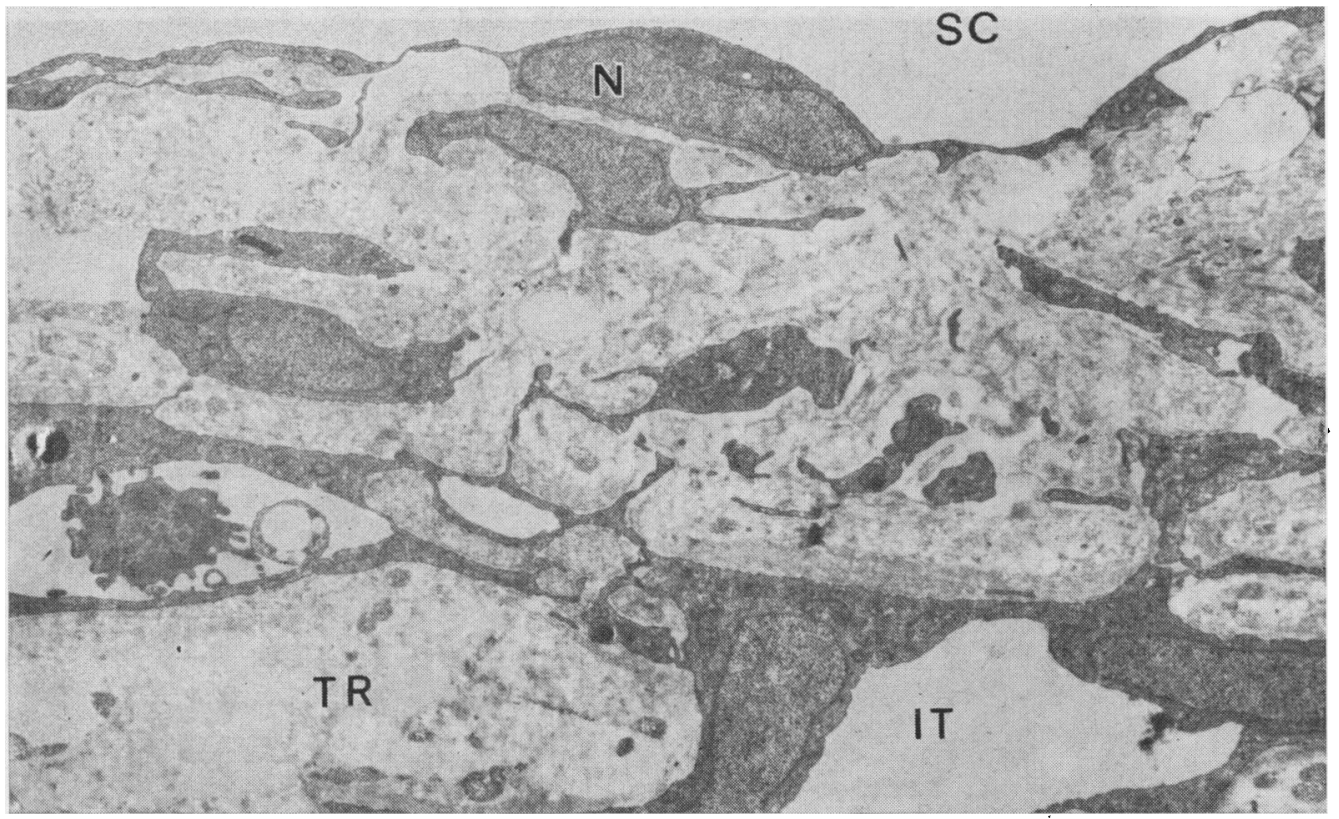

FIG. I3 Trabecular wall of Schlemm's canal (SC) in a moderately advanced case (aged 7o years) of simple glaucoma showing an attenuated endothelial lining with a flattened nucleus $(\mathcal{N})$ and an abundance of extracellular elements in the endothelial meshwork. $T R=$ Trabecular sheet. $I T=$ Intertrabecular space. Osmium fixed, Epon embedded, lead citrate stained. Electron micrograph. Approx. $\times 2,100$ 


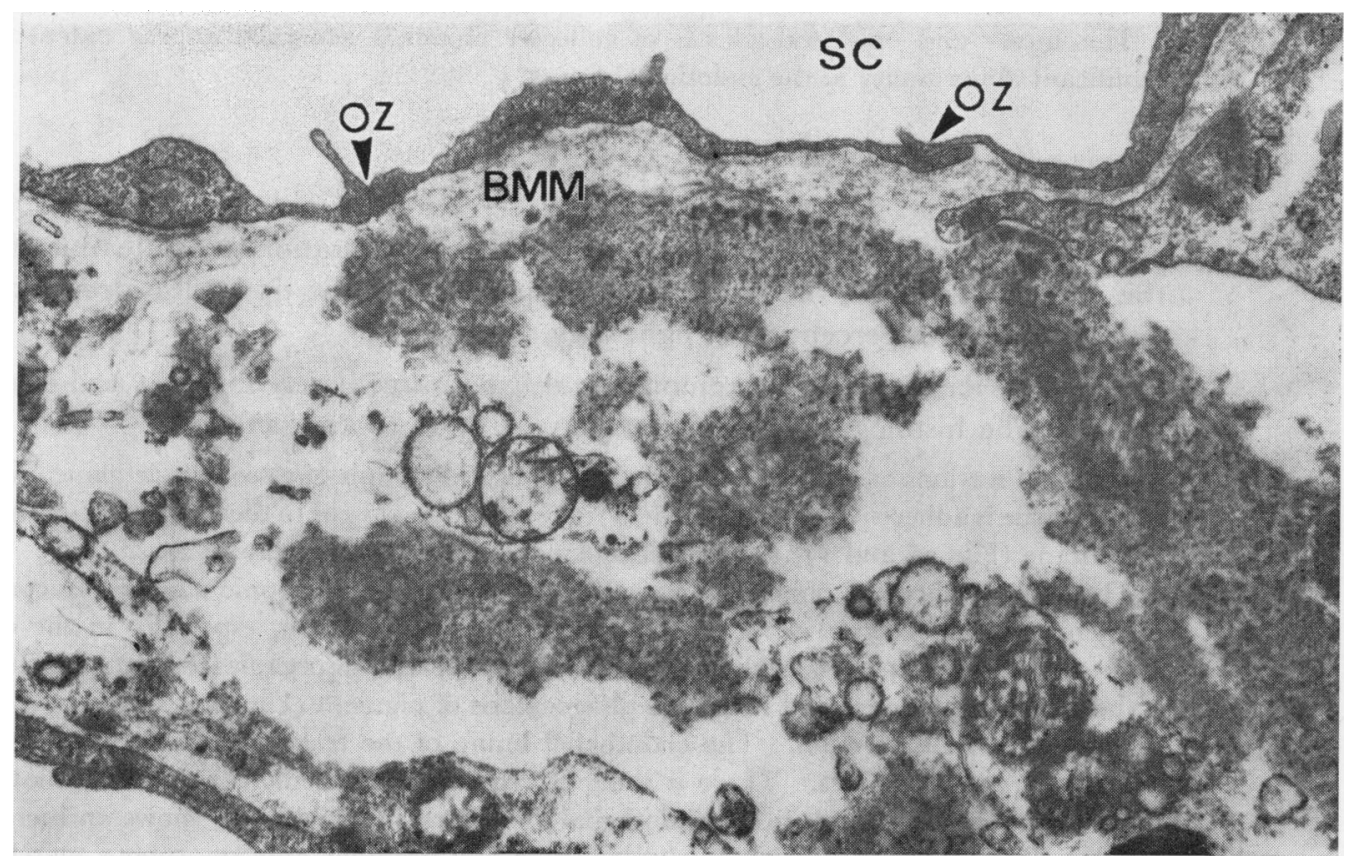

FIG. I4 Trabecular wall of Schlemm's canal $(S C)$ in advanced stage of simple glaucoma, showing attenuated endothelial lining, basement membrane material (BMM), granular and fusiform elements, and degenerating cells in adjacent area. $\mathrm{OZ}=$ Occluding zonule. Glutaraldehyde/osmium fixed, Araldite embedded, uranyl acetate and lead citrate stained. Electron micrograph. $\times 19,000$

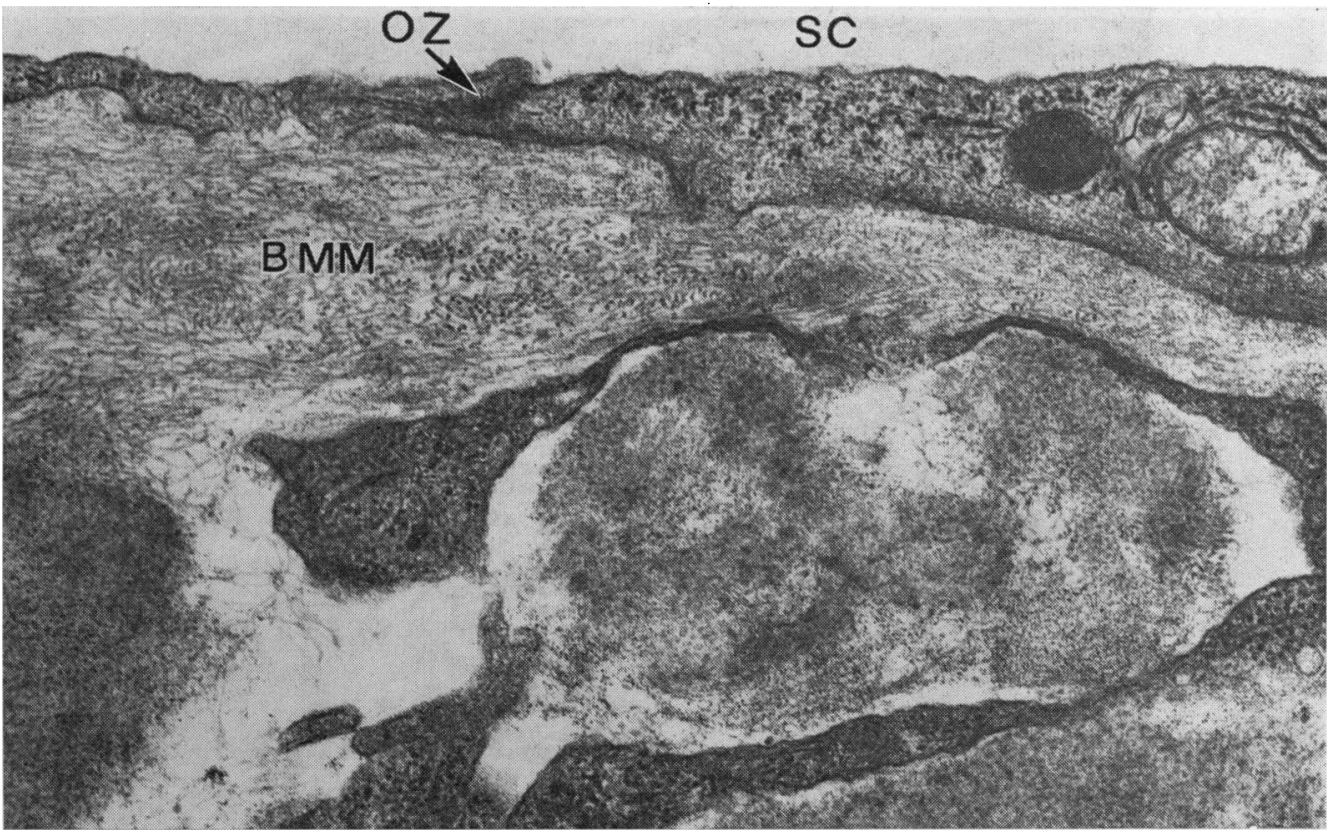

FIG. I5 In many places, the basement membrane material (BMM) beneath the trabecular wall of Schlemm's canal $(S C)$ is rich in microfibrils. Note also an abundance of granulo-fibrillar material in adjacent region. $O Z=$ Occluding zonules. Moderately advanced case of simple glaucoma, aged 70 years. Osmium fixed, Epon embedded, uranyl acetate and lead citrate stained. Electron micrograph. $\times 31,500$ 
The intra- and episcleral plexus of collector channels generally appear patent and show no significant abnormality in the endothelial lining.

GLOSED-ANGLE (ABSOLUTE) GLAUCOMA

The eye was enucleated surgically from a 4 I-year-old patient with closed-angle glaucoma after intermittent attacks of pain of nearly 12 months' duration. At the time of enucleation the cornea was clear, the ocular tension $40 \mathrm{~mm} . \mathrm{Hg}$, the optic disc deeply cupped, and vision reduced to perception of light only.

The anterior segment was removed and fixed immediately for light and electron microscopy. The histological and ultrastructural findings are as follows:

Meridional sections of the angle confirm the clinical diagnosis of closed-angle glaucoma; the root of the iris tissue is adherent to the inner uveal trabeculae except near to their termination into Descemet's membrane (Figs 16 and 17 ). The trabecular meshwork is relatively compact.

The other trabecular changes are similar to those seen in chronic simple glaucoma, i.e. slight thickening of the cortical zone, an increase in "curly" collagen, especially in the cortical zone, some degenerative changes in the collagen core, widespread disorganization and degeneration of the trabecular endothelium, and increased phagocytosis of pigment (Fig. 18).

Schlemm's canal is open. The endothelial lining of the trabecular wall shows an almost total absence of giant vacuoles. There is some increase in the basement membrane material beneath the endothelial lining and in the endothelial meshwork; the latter also shows an increase in extracellular elements, consisting of "curly" collagen and granular and amorphous material (Fig. 19). To a minor degree similar changes may be observed in the connective tissue zone of the corneoscleral wall. The intrascleral channels are patent and are filled with blood elements.

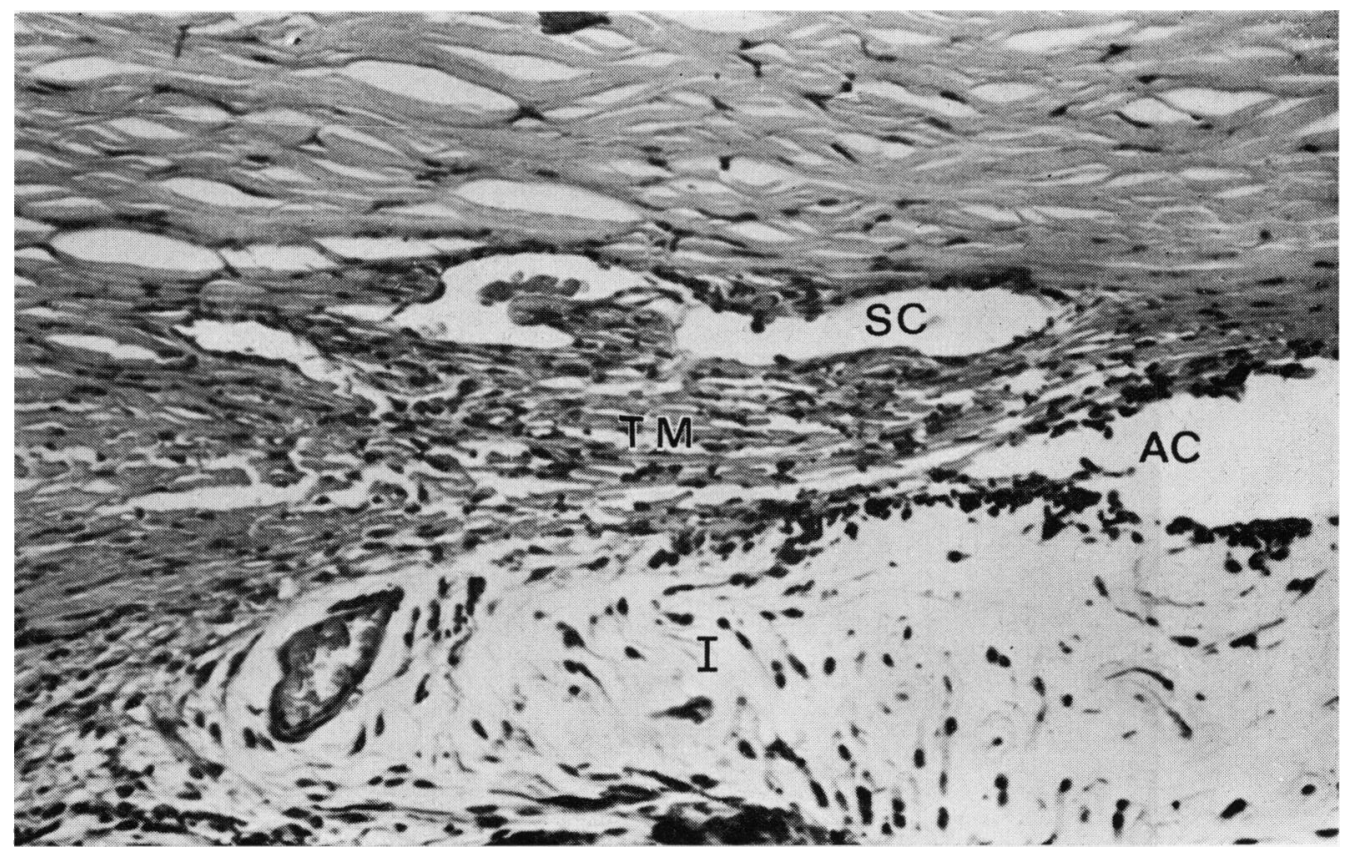

FIG. I6 Meridional section of angular region in a case of closed-angle glaucoma (41 years), showing almost total adherence of root of iris (I) with trabecular meshwork (TM); Schlemm's canal $(S C)$ is open. AC = Anterior chamber. Glutaraldehyde fixed, paraffin wax embedded, haematoxylin and eosin stained. Light photomicrograph. $\times 230$ 


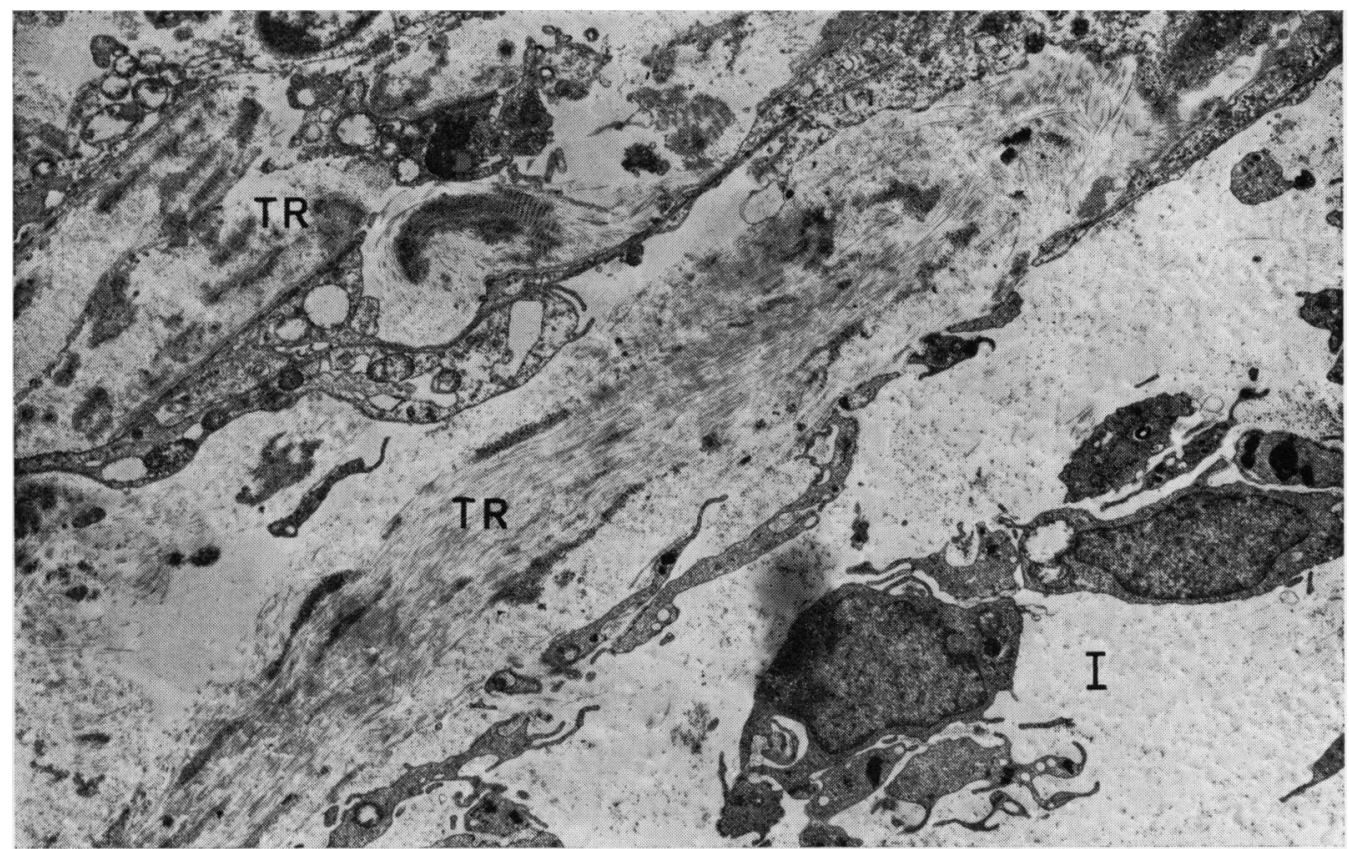

F1G. 17 Survey electron micrograph of fibrous adhesion of root of iris $(I)$ to uveal trabecular sheets (TR). Note disorganization in architecture of trabecular sheets. Glutaraldehyde/osmium fixed, Araldite embedded, uranyl acetate and lead citrate stained. Same case as Fig. $16 . \quad \times 4,350$

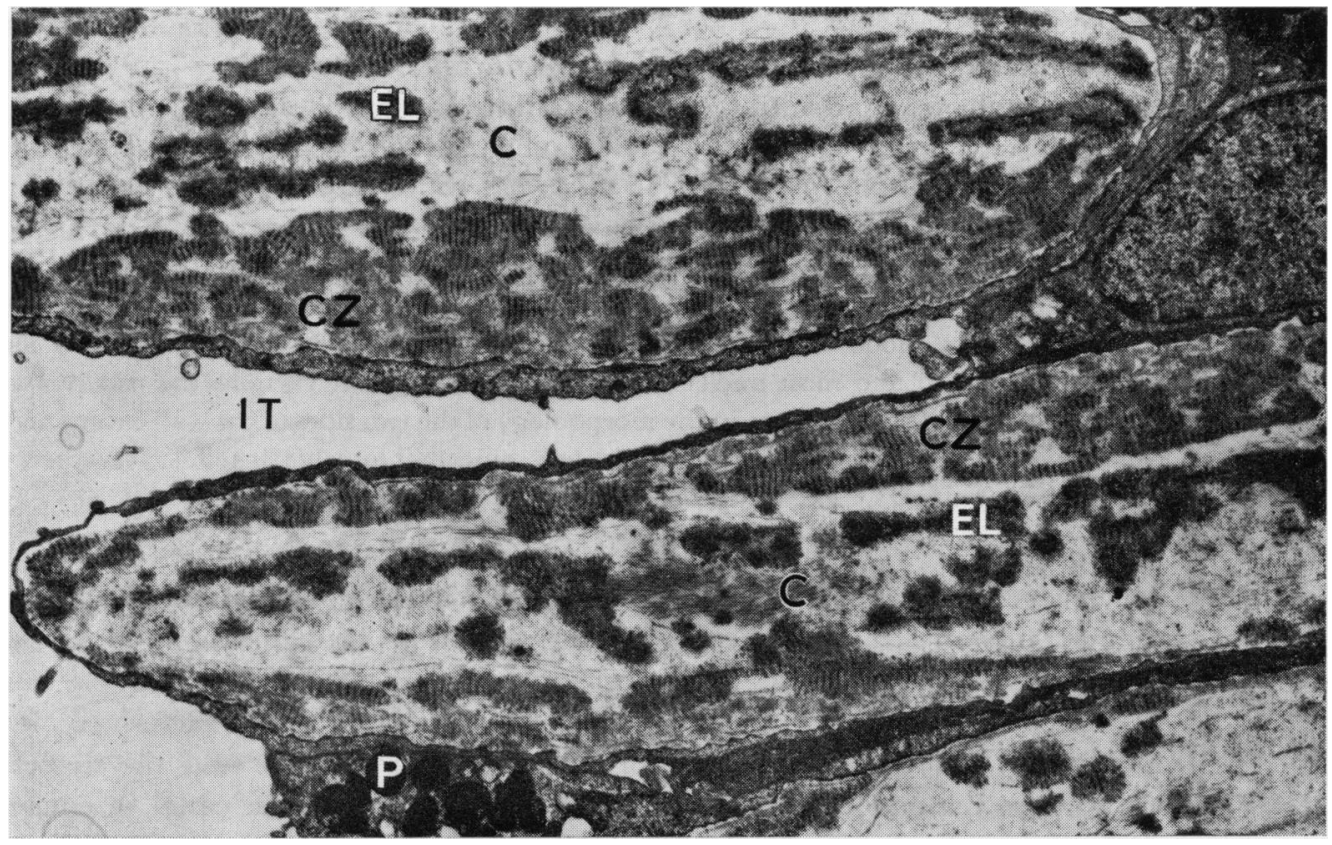

FIG. I8 Electron micrograph of trabecular sheets, showing thickened cortical zone $(C Z)$ and abundance of "curly" collagen. The central core is occupied by collagen fibrils $(C)$ and elastic element $(E L)$, the latler also being associated with "curly" collagen. Note also pigment granules $(P)$ in trabecular endothelium. IT = Intertrabecular space. Same case as Fig. $16 . \times 7,000$ 


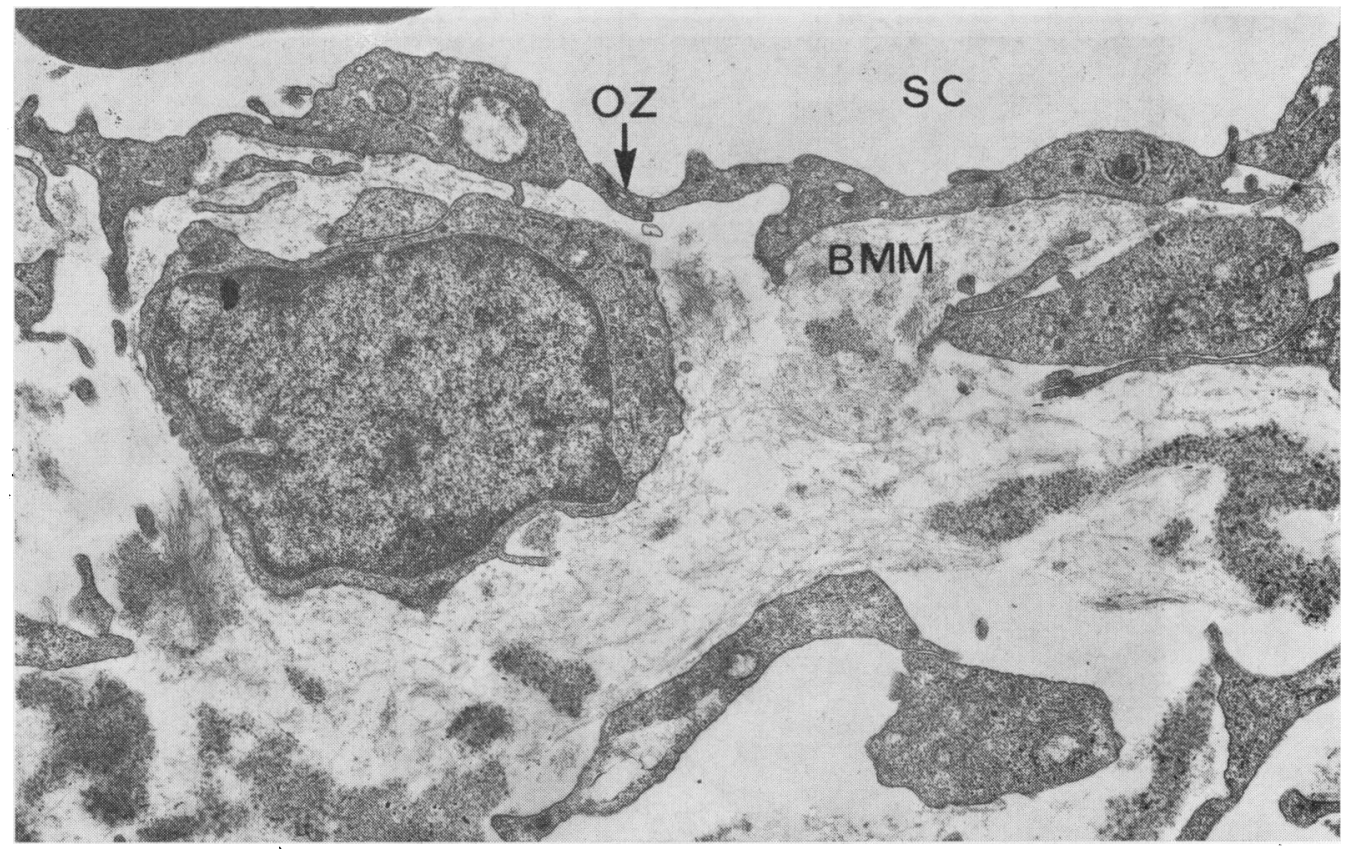

FIG. I9 Electron micrograph of trabecular wall of Schlemm's canal $(S C)$, showing intact endothelial lining devoid of giant vacuoles, basement membrane material (BMM), and scattered foci of granulo-amorphous and fusiform structures. $O Z=$ Occluding zonule. Same case as Fig. $16 . \quad \times 11,75^{0}$

\section{CONGENITAL GLAUCOMA}

Three trabeculectomy specimens removed surgically from cases in advanced stages of congenital glaucoma were studied by electron microscopy.

The histological and ultrastructural findings are as follows:

There ${ }^{*}$ is widespread structural disorganization and degenerative changes in the fibrous and cellular components of the trabecular meshwork with no evidence of a membrane covering the inner aspect of the trabecular tissue (Fig. 20).

The endothelial cells covering the trabecula are scanty; consequently, the typical architecture of individual trabecular sheets is difficult to recognize. The lumen of Schlemm's canal is patent, containing a few blood corpuscles and lined by a continuous endothelium. The individual cells appear attenuated and most nuclei are flattened. The giant vacuoles are totally absent (Fig. 2 I).

There is little or no difference in the morphology of the two sides of the wall except on the trabecular aspect where the supporting connective tissue is somewhat loosely packed. The adjacent intrascleral channels are patent.

\section{Discussion}

In view of the obvious limitations in acquiring material from glaucomatous patients suitable for ultrastructural studies and of the alterations brought about in the natural course of the disease by medical and surgical treatment, the findings in glaucomatous eyes presented here should be considered as indicative rather than conclusive. It is, however, interesting to note the similarity of the trabecular changes and the depletion of giant vacuoles in the endothelial cells of the trabecular wall of the canal in congenital, closed and open-angle glaucomas, despite the apparent differences in the clinical course of these conditions.

The structural alterations in the trabecular meshwork of the simple glaucomatous eyes presented here have also been observed by other workers and some have placed special 


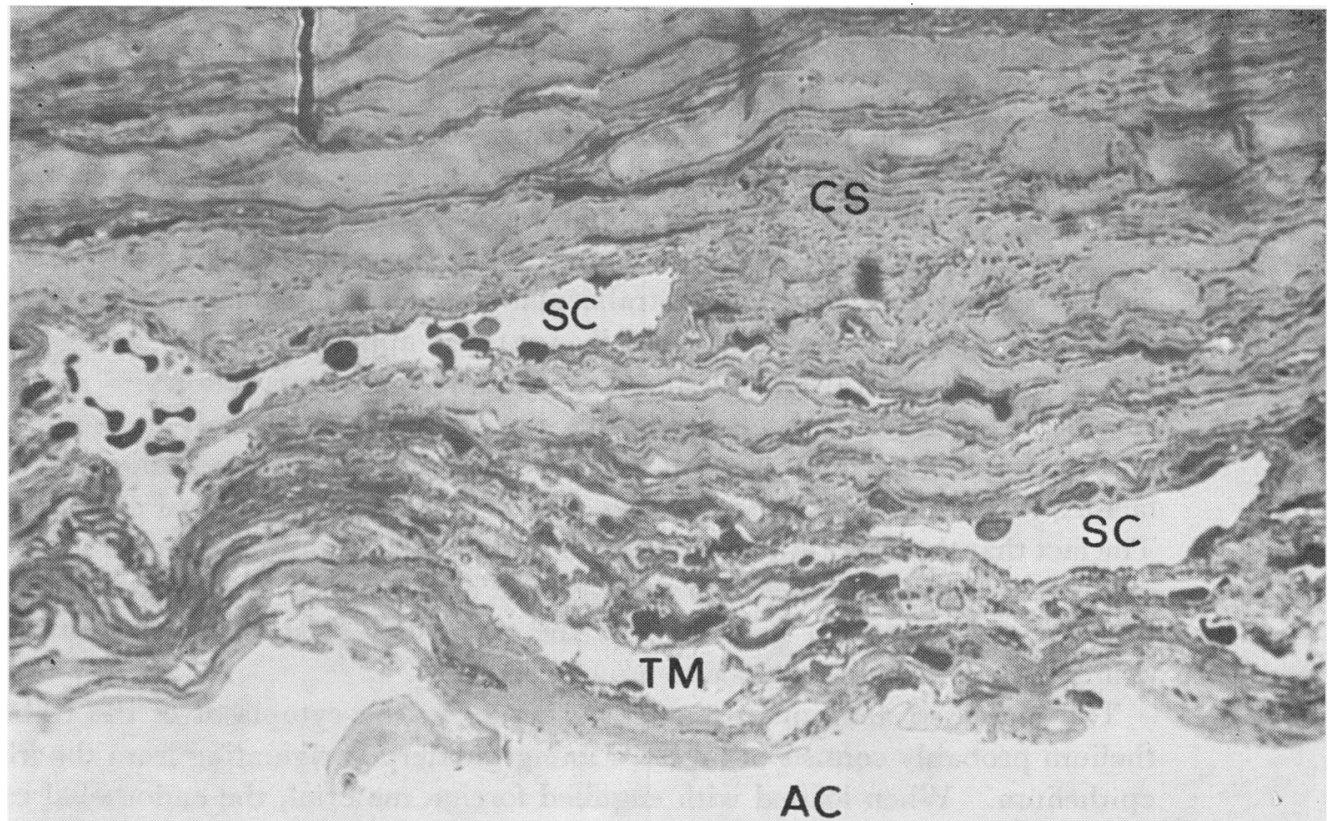

FIG. 20 Meridional section of a trabeculectomy specimen from an infant (aged 20 months) with congenital glaucoma, showing patent lumen of Schlemm's canal $(S C)$ and disorganized trabecular meshwork $(T M) . \quad A C=$ Anterior chamber. $C S=$ Corneo-sclera. Glutaraldehyde/osmium fixed, Araldite embedded, toluidine blue stained I $\mu$ thick section. $\times 560$

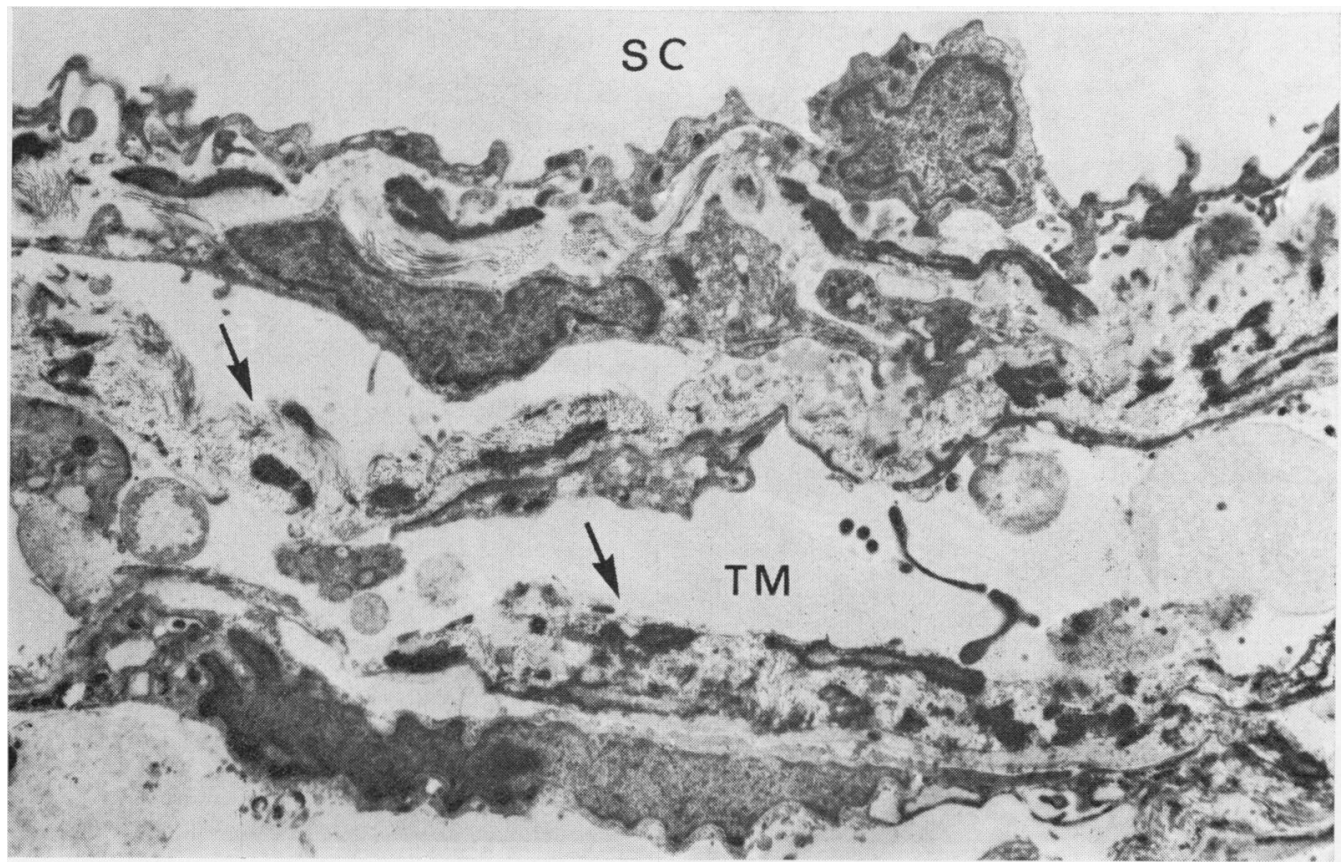

FIG. 2I Survey electron micrograph of the trabecular wall of Schlemm's canal (SC) and adjacent trabecular meshwork (TM) in congenital glaucoma. Note attenuated endothelial lining of trabecular wall devoid of giant vacuoles and disorganized trabecular meshwork, together with a loss of an endothelial covering in some places (arrowed). Glutaraldehyde/osmium fixed, Araldite embedded, uranyl acetate and lead citrate stained. $\quad \times 5,250$ 
emphasis on the formation of "curly" collagen (Garron, I959; Speakman, I96 I ; Speakman and Leeson, 1962, 1967; Holmberg, 1963; Boles-Carenini and Orzalesi, 1967; Rohen and Drecoll, I97I; and others). These findings in the trabecular meshwork, however, do not seem to be specific for simple glaucoma, since similar changes are present in the angle-closure (absolute) glaucoma reported here and have also been shown in secondary glaucomas of long standing by other workers (Rohen and Straub, 1967). In view of similar but less extensive trabecular changes in normotensive senile eyes (Fig. 22) (Tripathi, I969b; Rohen and Drecoll, I971), it appears that the formation of "curly" collagen in the trabecular sheets of simple glaucomatous eyes may not be a specific factor in the pathogenesis of this disease. It is more likely to be an ageing change and in simple glaucomatous eyes to be exaggerated by the mechanical and biochemical factors, including aqueous stagnation, that exist during the clinical course of this disease. The fact that the inter- and intratrabecular spaces in the present series of simple glaucomatous eyes are open and only slightly narrowed, even in advanced stages of the disease, seems to suggest that at least in these eyes the trabecular meshwork may not have been the primary site of total resistance to aqueous outflow.

The increased amount of pigment granules in the cytoplasm of the trabecular endothelium probably consists of ingested foreign material originating from the iris and ciliary epithelium. When loaded with engulfed foreign material, the endothelial cells probably separate from the trabecular sheets and assume the role of wandering histiocytes (Rohen and Zypen, 1968; Tripathi, I969b). This process would seem to explain the greater tendency of the trabecular sheets to be devoid of endothelial covering. The fibrous components of these trabecular sheets thus come into direct contact with the aqueous

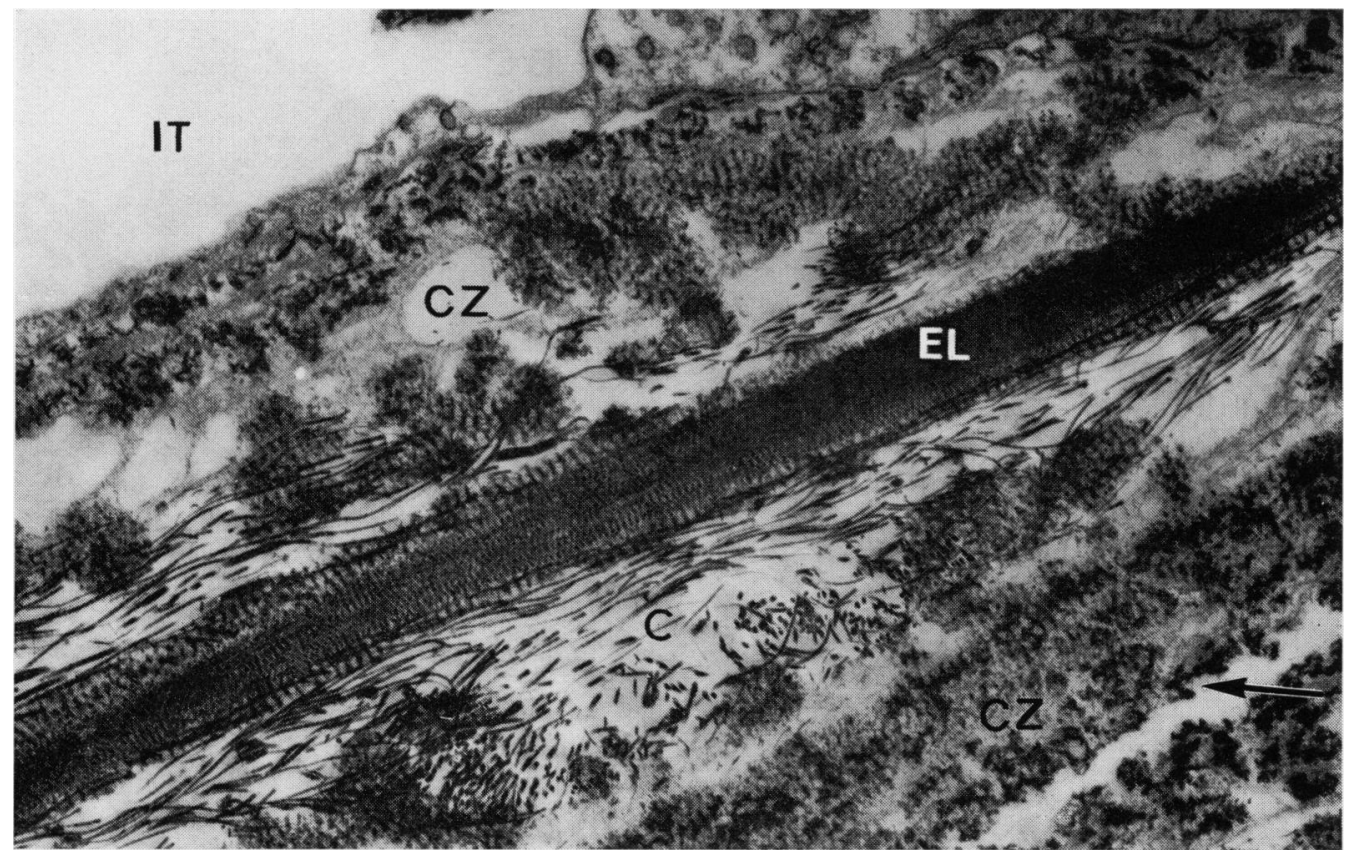

FIG. 22 Trabecular sheet in a normotensive eye (80 years), showing thickened cortical zone $(C Z)$ with abundance of "curly" collagen, collagen fibrils $(C)$ in central core, and elastic element $(E L)$ in association with "curly" collagen. Arrow denotes absence of endothelial covering of trabecular sheet. IT = Intertrabecular space. Osmium fixed, Araldite embedded, phosphotungstic acid stained. Electron micrograph. $\quad \times 14,700$ 
humour and this may contribute to the degenerative changes seen in the fibrous components of some trabecular sheets.

The observed changes in the region of the trabecular meshwork of congenital glaucoma reported here do not allow far-reaching conclusions. It is possible, as some workers suggest (Barkan, 1942; Shaffer, 1967; Manschot, 1970), that the meshwork remains embryonic and that differentiation into individual trabecular sheets fails to take place. Alternatively, this may be the consequence of increased tension and aqueous stagnation (Manschot, 1970). The significance of the structural alterations in the wall of Schlemm's canal, including the loss of endothelial vacuolation, in the pathogenesis of this disease remains to be clarified.

In view of the role of the endothelial vacuolation in the outflow of the aqueous humour in normotensive mammalian eyes discussed earlier, a quantitative and qualitative depletion of the vacuoles in the endothelial lining of Schlemm's canal in the glaucomatous eyes reported here would seem to be the most significant change. While the site of obstruction to aqueous outflow in chronic simple and congenital glaucomas is still debatable, there is little doubt as to the nature of obstruction to aqueous outflow in acute glaucoma, since the adhesion of the root of the iris to the trabecular meshwork is an established finding. If endothelial vacuolation is dependent on the feed-back mechanism of the aqueous humour, then the depletion of vacuoles in the latter case could easily be explained as a secondary change.

In chronic simple glaucoma, at least in the cases studied, the structural alterations in the trabecular wall of Schlemm's canal suggest it to be the main site of resistance to outflow. The functional nature of the block, however, remains a matter for speculation. It is possible that the block is purely mechanical in nature, produced by the build-up of the fibrous, granulo-amorphous, and basement membrane material in this region. The presence of similar, but less extensive, extracellular elements in normotensive eyes (Fig. 23) suggests, however, that this structural alteration in glaucomatous eyes is probably no more than an exaggerated senile and degenerative change which, in some cases, may be capable of preventing the aqueous from reaching the endothelial lining, and may consequently lead to the loss of vacuolation.

Alternatively, the primary block may lie in the malfunctioning of the endothelial lining of the canal (possibly due to enzymatic, nutritional, or immunological factors). If the endothelial vacuolation (termed macropinocytosis) is in fact a dynamic process responsible for the normal aqueous outflow, then the fault is most likely to occur in the initial stages of the vacuolation cycle. This could result from a gradual increase in the resistance offered by the cell membrane and/or a gradual loss of cell activity. In some cases it may perhaps be related to a genetic defect, to an ageing process, or to abiotrophy, while in others it may be due to altered aqueous content or degenerative products originating from the angular tissue. Clearly, the answer to many of these questions is to be found in a greater understanding of the factors responsible for the control of the process of endothelial vacuolation.

It is to be remembered, however, that while clinically chronic simple glaucoma can be considered as a distinct entity, the aetiopathogenesis of this symptom complex may be diverse and the emphasis of pathology in the exit pathway of the aqueous may vary from one region to another.

My thanks are due to Prof. Norman Ashton, F.R.S., for his helpful comments and to my wife and co-worker Dr. Brenda Tripathi for her enthusiastic assistance.

It is a pleasure to acknowledge the financial support of the Medical Research Council in this project. 


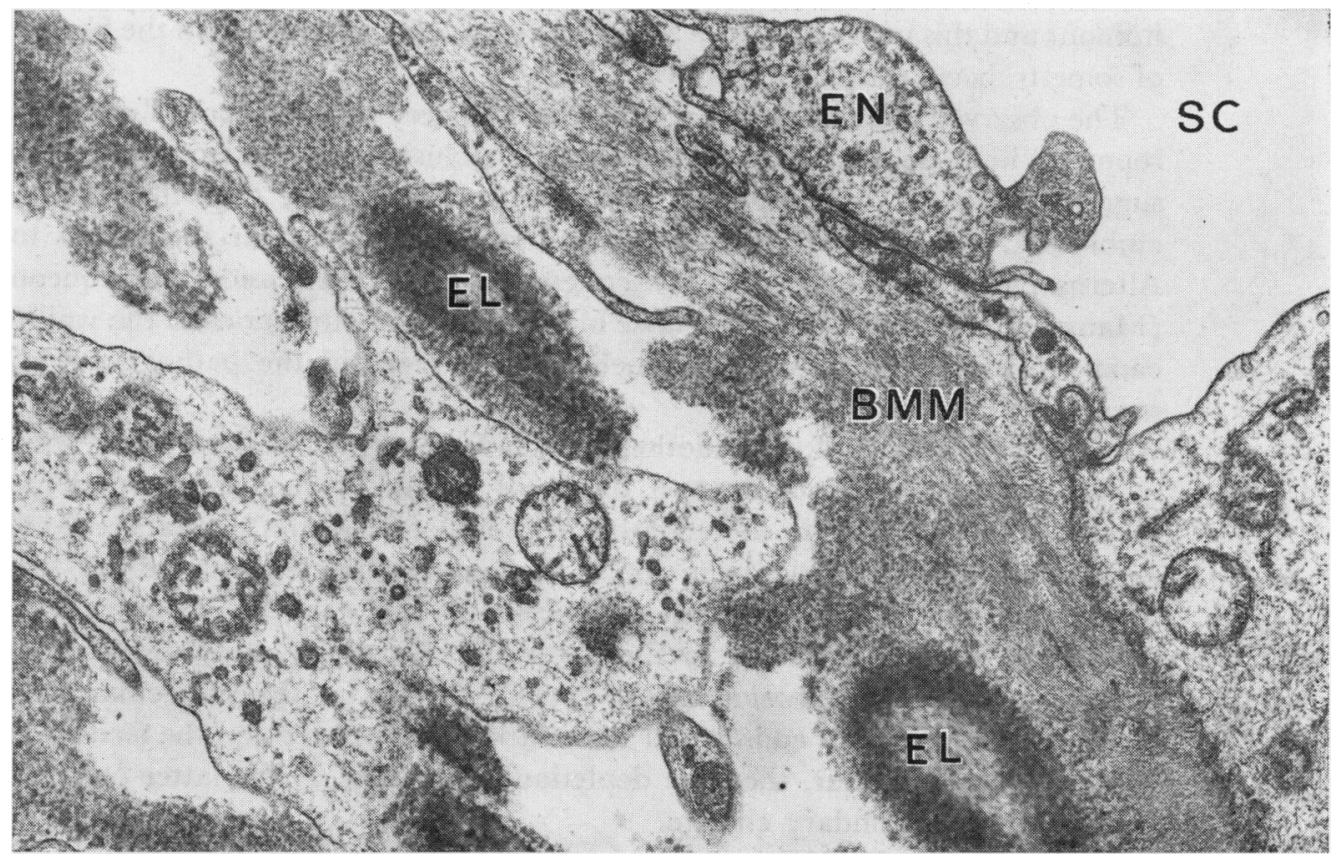

FIG. 23 Trabecular wall of Schlemm's canal (SC) in normotensive eye (8o years), showing healthy endothelial lining $(E \mathcal{N})$, microfibrils in basement membrane material $(B M M)$, and granulo-fibrillar and fusiform elements. Note also elastic element $(E L)$ in association with "curly" collagen. Same case as Fig. $22 . \quad \times 33,500$

\section{GOMMENTARY}

TRABECULAR GHANGES IN GLAUGOMA

(I) The function of the vacuolated cells in the endothelial lining of Schlemm's canal

It is felt that the transport of fluid by these vacuoles may be initially an active process requiring metabolic energy, but this later becomes pressure-dependent. The cells, however, contain only a moderate amount of energy-producing organelles, such as mitochondria, compared to ciliary epithelium or corneal endothelium and probably have no more of these organelles than the cells of the trabecular sheets. It is therefore extremely unlikely that they will actively transport a vacuole 2-15 $\mu \mathrm{m}$. in diameter filled with water or particulate matter. The analogy was used of blowing up a balloon, where the initial expansion requires a great deal of energy but from there onwards very little energy is needed to blow the balloon up to its fullest extent. The process of macropinocytosis of the endothelial cells of the trabecular wall allows infolding of the cell membrane. The pressure gradient across the trabecular wall of Schlemm's canal is about $5^{-7} \mathrm{~mm}$. $\mathrm{Hg}$ and may be sufficient to allow the vacuole to increase in size and complete its cycle. If the trabecular apparatus is normal and the hydrostatic pressure inside the anterior chamber is increased, theoretically the cells must function more rapidly, by increasing the number and turnover rate of vacuoles, and/or the period when there is an intracellular channel must be sustained in order to allow more fluid to pass into Schlemm's canal, restoring the intraocular pressure to normal. The diminished number of vacuoles found in cases of simple glaucoma could be a secondary phenomenon, particularly as similar changes were found in trabecular specimens from cases of congenital and closed-angle glaucoma.

\section{(2) The effect of pilocarpine on the vacuolated cells}

There is no satisfactory experimental evidence to confirm the thought that the number of vacuolated cells is reduced by pilocarpine but, if pilocarpine does in fact affect the vacuolated cells, this must be a direct action of the drug as these cells are apparently devoid of nerve endings. 